\title{
Shock Response Spectra Reconstruction of Pointwise Explosive-Induced Pyroshock Based on Signal Processing of Laser Shocks
}

\author{
S. Y. Chong, ${ }^{1}$ J. R. Lee, ${ }^{1,2,3}$ and C. W. Kong ${ }^{2}$ \\ ${ }^{1}$ Department of Aerospace Engineering and LANL-CBNU Engineering Institute Korea, Chonbuk National University, \\ 567 Baekje-daero, Deokjin-gu, Jeonbuk, Jeonju 561-756, Republic of Korea \\ ${ }^{2}$ Structures and Materials Department, Korea Aerospace Research Institute, Daejeon 305-333, Republic of Korea \\ ${ }^{3}$ X-NDT Inc., \#304, Building of Engineering Institute Korea, 567 Baekje-daero, Deokjin-gu, Jeonbuk, Jeonju 561-756, Republic of Korea
}

Correspondence should be addressed to J. R. Lee; leejrr@jbnu.ac.kr

Received 6 December 2012; Accepted 14 March 2013; Published 3 April 2014

Academic Editor: Gyuhae Park

Copyright (C) 2014 S. Y. Chong et al. This is an open access article distributed under the Creative Commons Attribution License, which permits unrestricted use, distribution, and reproduction in any medium, provided the original work is properly cited.

Pyroshock has been an issue of great concern for aerospace and defense industrial applications. When pyroshock devices are detonated, they can easily cause failures in electronic, optical, relay, and magnetic components generally in mid- and far-fields which is not avoidable at the design level. Thus, many numerical and experimental pyroshock simulations have been widely studied to predict explosive-induced pyroshock effect quantitatively, especially the shock response spectrum (SRS). In this study, a laser shock-based pyroshock reconstruction method is proposed to simulate a pointwise explosive-induced pyroshock signal. The signal processing algorithm for the laser shock-based pyroshock reconstruction is developed in a LabVIEW platform and consists of subbands decomposition, SRS matching in decomposed bands, and wave synthesizing. Then, two experimental setups are configured to obtain pyroshock signals and laser shock signals at four points in an aluminum plate. The reconstructed pyroshock signals synthesized according to the signal processing of the laser shocks demonstrate high similarity to the real pyroshock signals, where the similarity is evaluated by the mean acceleration difference between the SRS curves. The optimized settings of the subband decomposition were obtained and can be in the future used in a pyroshock simulator based on laser shock for pyroshock simulation at any arbitrary point.

\section{Introduction}

Pyrotechnic shock or pyroshock has been an issue of great concern for aerospace and defense industrial applications. Pyroshock is the transient oscillatory response of a structure to loading (high frequency and high magnitude stress waves) induced by the detonation of pyrotechnic devices using pointwise or linear explosives [1], incorporated into or attached to the structure. As a result, its high frequency energy can easily cause failures in electronic, optical, relay, and magnetic components, especially in aerospace applications during the separation of structural subsystems, the deployment of appendages, and the activation or deactivation of subsystems [2].

Various pyroshock simulation methods, which are generally classified as experimental and numerical simulations, have been studied to predict pyroshock effects quantitatively on the intended pyroshock environments. These pyroshock environments can be classified as near-, mid-, and far-field environments $[1,3]$. Then, a shock response spectrum (SRS) is used as a tool to analyze and quantify a pyroshock. The SRS is a graphical representation of maximum response regarding a single response for a single degree of freedom (SDOF) system as a function of the natural frequency of the SDOF [2]. Various experimental test simulations have been introduced, and these include ordnance test, scaled test, electrodynamic shaker tests, and tuned or tunable resonant fixtures, resulting in realistic pyroshock data with high amplitude and high frequency [1]. Generally, the preparation of the specimens and sensors is often problematic and the experimental repeatability is poor. In addition, the experimental methods are of high cost because many sample structures or realistic 
models are used in pyroshock study. In numerical simulation methods, they are used to complement experimental simulation and shorten the overall design process, and they include finite element method (FEM), statistical energy analysis (SEA), hybrid methods, and hyrocode methods [1]. However, numerical methods show inefficiency in pyroshock wave analysis when insufficient elements are used [4].

From the past decades, laser excitation has been used for various applications such as material property measurements and structural nondestructive testing and evaluation and for the simulation of elastic waves [5]. Recently, Lee et al. [1] have shown the feasibility of a laser excitation for pyroshock simulation. In that study, it was reported that the laser excitation was able to be manipulated to be similar to the mechanical shock with a peak-to-peak acceleration of $7000 \mathrm{~g}$ and a central frequency of $70 \mathrm{kHz}$.

Many signal processing techniques have been introduced in the field of pyroshock. Recently, Brake [6] developed a method to reconstruct a time-domain wave from the SRS when only SRS is available. This method used three theoretical basic functions to reconstruct a time history that corresponds to a given SRS: an impulse function, a sine function/damped sine function, and a modified Morlet wavelet. However, a high fidelity representation of the SRS was needed, and it required significantly more time to obtain accuracy in matching the target SRS when the genetic algorithm approach was used. Hale and Adhami [7] researched for synthesis of SRS using the time-frequency analysis. Besides the techniques introduced in pyroshock, signal decomposition and synthesis methods [8-10] have been widely used. Particularly, the subbands decomposition method based on filter bank has been used not only for data compression [9] but also for feature extraction [8]. The work principle is that a filter bank contains a set of analysis filters which decompose the bandwidth of the input signal into subband signals with uniform frequency bands. Then, processing can be performed on the subbands according to a specific application [8].

In regard to the prior studies, the laser excitation, which has nondestructive property, can provide better solutions than the conventional experimental techniques, where the laser excitation will not damage the real structures or will not alter the properties of the target structure. Thus, the simulator based on laser excitations can be applied even to the flightready structures. Moreover, the laser excitation is also able to obtain real stress wave from the real target structure for pyroshock simulation. With these advancements of laser excitation, a reconstruction method of shock response spectra (SRSs) for pointwise explosive-induced pyroshock based on the signal process of laser shocks is first proposed in this paper. In addition, the finding of this work is to determine the optimized parameter settings of the proposed signal processing algorithm. Then, the settings will be further used for a new pyroshock simulator development. The simulator will be incorporated with the proposed signal processing algorithm, which can simulate a pyroshock signal using a laser shock signal at any arbitrary point with no requirement of any real pyroshock signal. Two experimental setups are configured to obtain the pyroshock signal and the laser shock signal. In addition, the signal processing algorithm is developed and realized in a LabVIEW platform with two main functions, subbands decomposition and SRS matching synthesis. In subbands decomposition, both pyroshock and laser shock signals are decomposed into the subbands using a set of hieratical bandpass filters. Then, the decomposed signals are inputted into the synthesis process with the SRS matching function to reconstruct the SRS of the pyroshock signal based on the laser shock signal. The laser shock signal matched by the proposed algorithm is considered as a reconstructed pyroshock signal. Then, the similarity of a reconstructed pyroshock is analyzed by minimizing the acceleration difference with the SRS of the real pyroshock.

\section{Pyroshock Shock Response Spectrum Reconstruction Algorithm Using Laser Shock}

Figure 1 shows the schematic diagram of the signal processing algorithm developed to reconstruct a pointwise explosiveinduced pyroshock using a laser shock. In order to realize this algorithm, first we assumed that pyroshock signals obey the wave superposition principle [11]. As shown in Figure 1, the overall algorithm consisted of two main functions, subbands decomposition and synthesis with the SRS matching function. In the subbands decomposition, the Butterworth infinite-impulse-response (IIR) bandpass filter was used to decompose both pyroshock and laser shock signals into a set of time-domain signals with different frequency bands. Commonly, SRS curve is plotted on a log-log graph where the log-scale $x$-axis is representing the natural frequency of a pyroshock and each cycle of the logarithmic scale covers a range of values spanning one factor of 10 . It also meant that the next cycle covers a range 10 times larger. In addition, a pyroshock is normally analyzed at the broad natural frequency range, for example, from $100 \mathrm{~Hz}$ to $100 \mathrm{kHz}$. For that, the appropriate selection of the number of subband filters used is a must in order to achieve narrow band in each subband signal. For example, 100-subband filters are selected to decompose the pyroshock signal $(100 \mathrm{~Hz}$ to $100 \mathrm{kHz}$ ), and each subband signal has equal bandwidth of $999 \mathrm{~Hz}$, respectively. With this decomposition bandwidth, it is difficult to obtain good analysis results in the lower natural frequency range of a pyroshock. Thus, to increase higher number of subband filters to obtain higher frequency resolution is not an appropriate way because it will cause the increase of processing time. In regard to this point, two levels of the subbands decomposition process were employed and denoted as Level 1 (L1) and Level 2 (L2). L1 was used to decompose the pyroshock signal with wider frequency bands accordingly to the natural frequency ranges set in each logscale cycle, respectively, and L2 was further used to decompose each subband signal of L1 with narrow frequency bands, respectively. Then, the SRS matching synthesis extracted features for SRS matching from the decomposed pyroshock and laser shock signals and synthesized the decomposed signals to obtain a reconstructed pyroshock signal. 


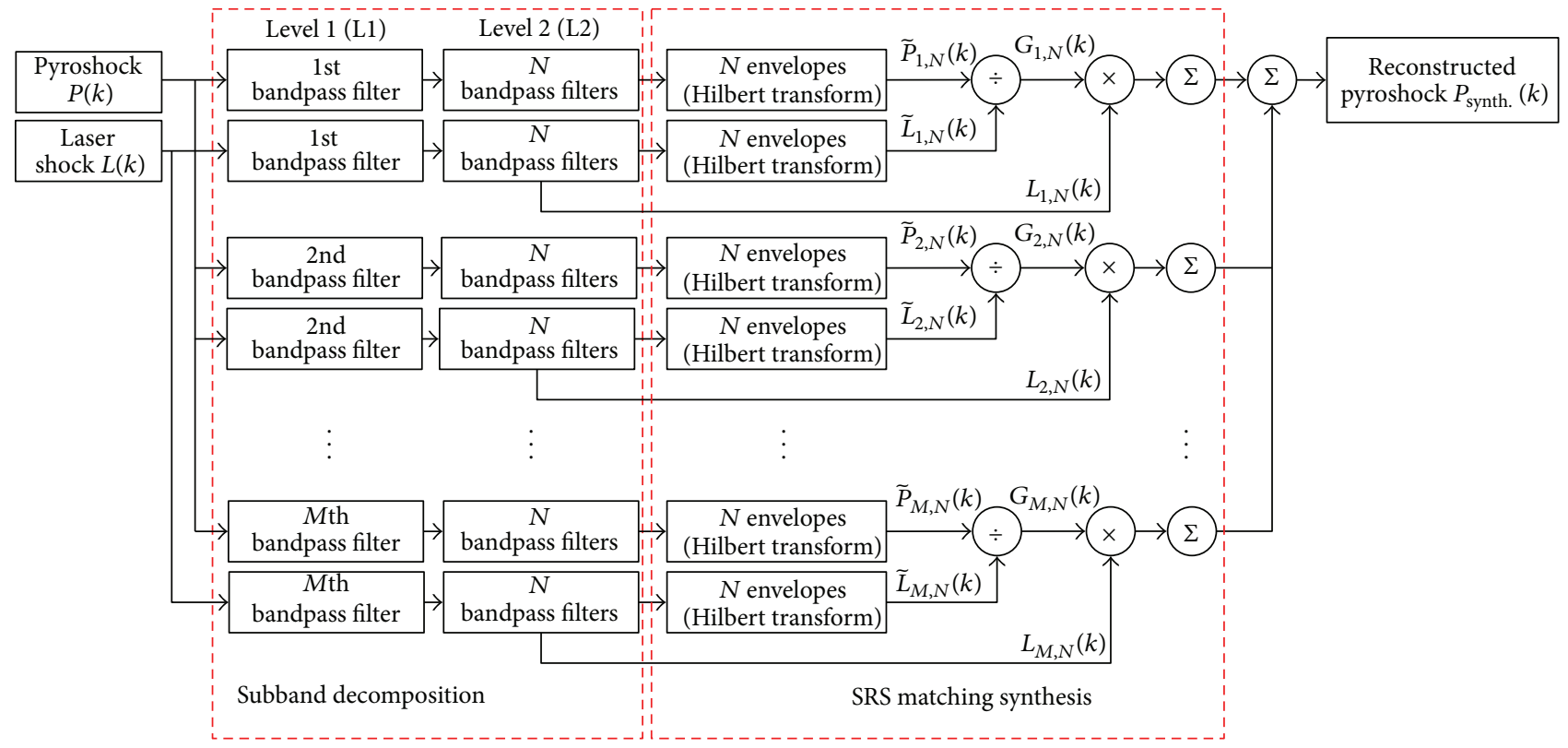

FIGURE 1: Schematic diagram of the signal processing algorithm to reconstruct a pyroshock signal using a laser shock signal.

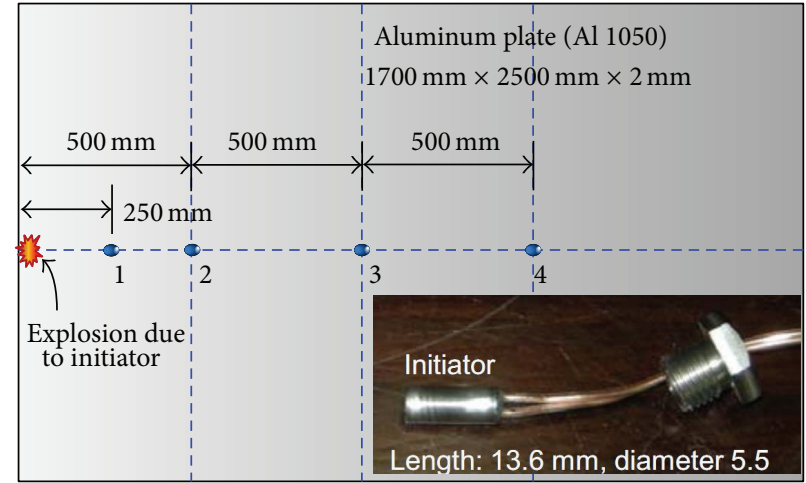

(a)

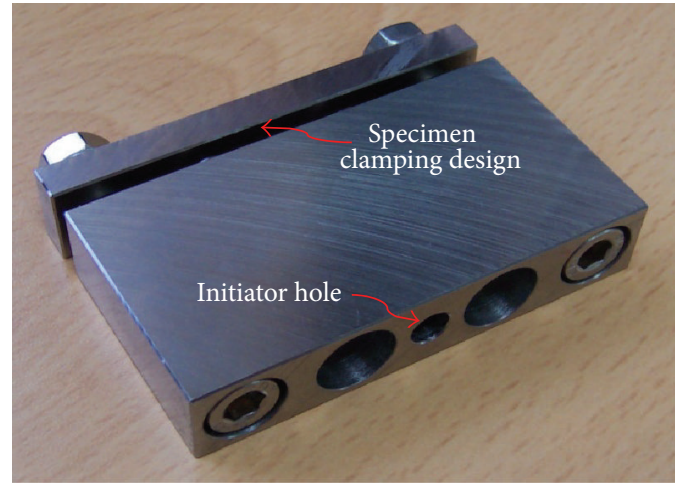

(b)

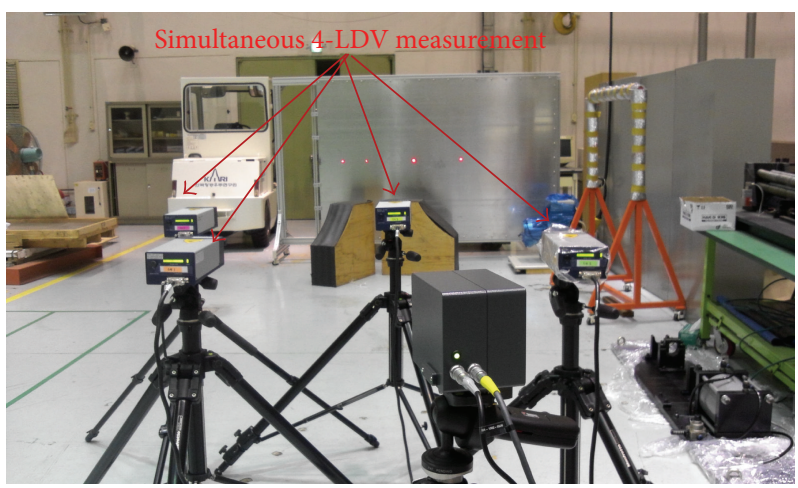

(c)

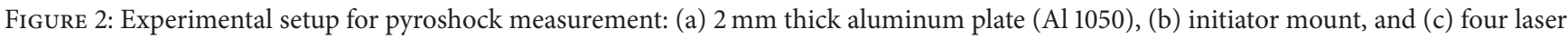
Doppler vibrometers setup configuration. 
TABLE 1: Examples of $M$ and $N$ bandpass filter matrix setting in Level 1 and Level 2.

Level 1 (L1)

$N$ bandpass filters

1

3

\begin{tabular}{lcccc} 
& $100-100 \mathrm{kHz}$ & $100-1 \mathrm{kHz}$ & $1 \mathrm{kHz}-10 \mathrm{kHz}$ & $10 \mathrm{kHz}-100 \mathrm{kHz}$ \\
\hline Level 2(L2) & & & & \\
$M$ bandpass filters & & & & \\
1 & $99.9 \mathrm{kHz}$ & $900 \mathrm{~Hz}$ & $9.0 \mathrm{kHz}$ & $90.0 \mathrm{kHz}$ \\
5 & - & $180 \mathrm{~Hz}$ & $9.8 \mathrm{kHz}$ & $18.0 \mathrm{kHz}$ \\
10 & - & $90 \mathrm{~Hz}$ & $900 \mathrm{~Hz}$ & $9.0 \mathrm{kHz}$ \\
20 & - & $45 \mathrm{~Hz}$ & $450 \mathrm{~Hz}$ & $4.5 \mathrm{kHz}$ \\
\hline
\end{tabular}

TABLE 2: Mean acceleration differences (\%) in the pyroshock SRS repeatability test over the four points.

\begin{tabular}{|c|c|c|c|c|}
\hline \multirow{2}{*}{ Case } & \multicolumn{4}{|c|}{ Mean acceleration difference (\%) } \\
\hline & Pt1 & Pt2 & $\mathrm{Pt} 3$ & Pt4 \\
\hline Ref-1 & 15.0 & 11.5 & 29.5 & 34.8 \\
\hline Ref-2 & 10.9 & 8.0 & 25.3 & 27.2 \\
\hline Ref-3 & 10.3 & 8.0 & 26.5 & 28.1 \\
\hline Average & 12.1 & 9.2 & 27.1 & 30.0 \\
\hline Average Pt1 Pt4 & \multicolumn{4}{|c|}{$19.6 \%$} \\
\hline
\end{tabular}

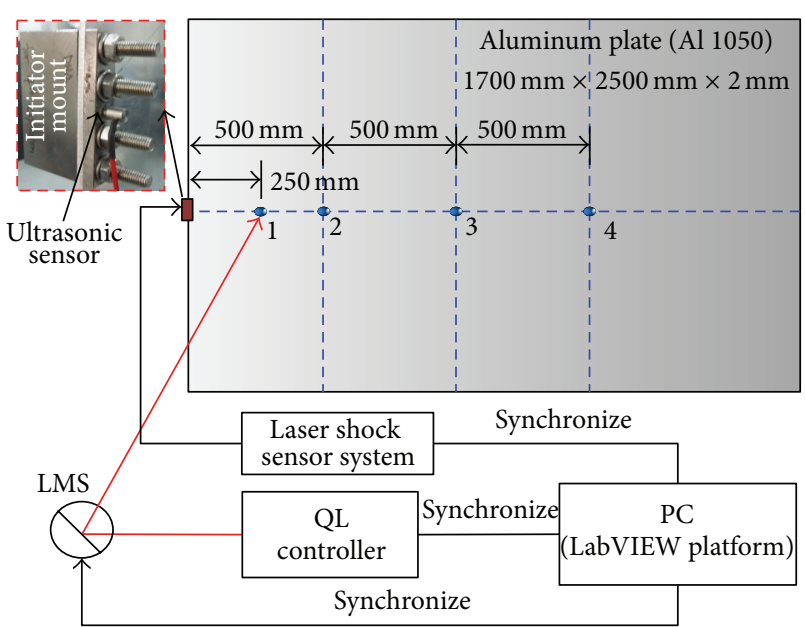

LMS: laser mirror scanner

QL: Q-switched continuous wave laser

FIGURE 3: Experiment setup for laser shock measurement in the same specimen in the pyroshock measurement.

As for the details of the proposed algorithm, a pyroshock signal and a laser shock signal, which are measured at the same local point, are inputted into the subbands decomposition. The pyroshock signal, denoted by $P(k)$, is used as a reference for the laser shock signal, denoted by $L(k)$, to convert the laser shock into a reconstructed pyroshock signal. Then, the notation of $k$ is the index of the discretetime instants of the signal. In L1, $P(k)$ is decomposed into different frequency bands by $M$ bandpass filters, as shown in Figure 1. At the same time, the decomposition process of $L(k)$ is also the same as the decomposition process of $P(k)$, and the decomposed signals of $L(k)$ and $P(k)$ are defined as $L_{i}(k)$ and $P_{i}(k)$, respectively, where the index is $i=1,2, \ldots, M$.

Once the L1 decomposition process is completed, both $P_{i}(k)$ and $L_{i}(k)$ are further processed in $\mathrm{L} 2$. The respective $i$ th decomposed signals of $P_{i}(k)$ and $L_{i}(k)$ are decomposed again into $N$ signals with equal bandwidths by $N$ bandpass filters, respectively. For example, as shown in Figure $1, P_{1}(k)$ is decomposed into $N$ signals and $P_{2}(k)$ is decomposed into another $N$ signals with $N$ bandpass filters, respectively. This process is also performed the same for $L_{1}(k)$. Then, the resultants of the two-level subbands decomposition process are defined as $P_{i, j}(k)$ and $L_{i, j}(k)$ for the pyroshock and laser shock signals, respectively, where the index is $j=1,2, \ldots, N$.

After the decomposition, the SRS matching and synthesis process with $P_{i, j}(k)$ and $L_{i, j}(k)$ follows. In this process, the signal envelope extraction function based on the Hilbert transform is used, and the function can be expressed as [12]

$$
\begin{aligned}
& E(t)=\sqrt{x(t)^{2}+H[x(t)]^{2}}, \\
& H[x(t)]=\frac{1}{\pi} \int_{-\infty}^{\infty} \frac{x(\tau)}{\tau-1} d \tau,
\end{aligned}
$$

where $E(t)$ is the enveloped signal of an input signal $x(t)$ and $H[x(t)]$ is the Hilbert transform of the input signal $x(t)$. Hence, based on (1), the total enveloped signals of $M \times N$ for each $P_{i, j}(k)$ and $L_{i, j}(k)$ are extracted and defined as $\widetilde{P}_{i, j}(k)$ and $\widetilde{L}_{i, j}(k)$, respectively, as shown in Figure 1. Once the enveloped 


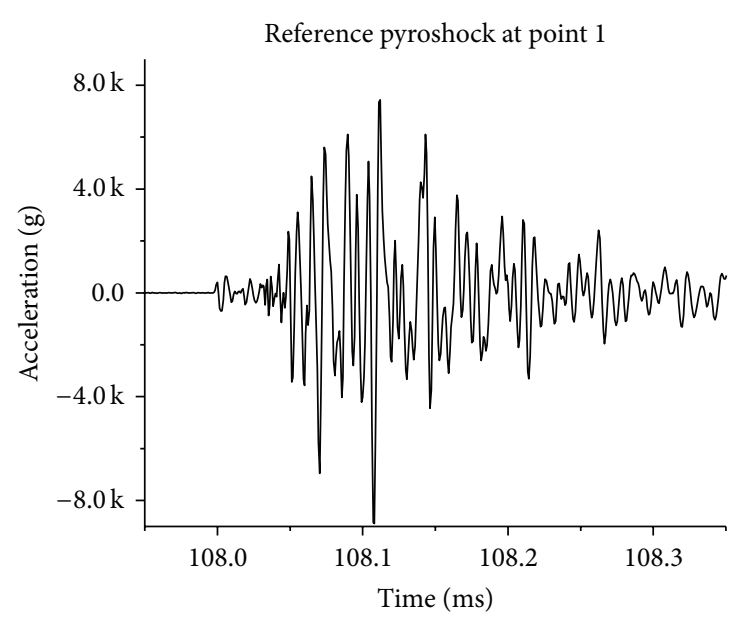

(a)

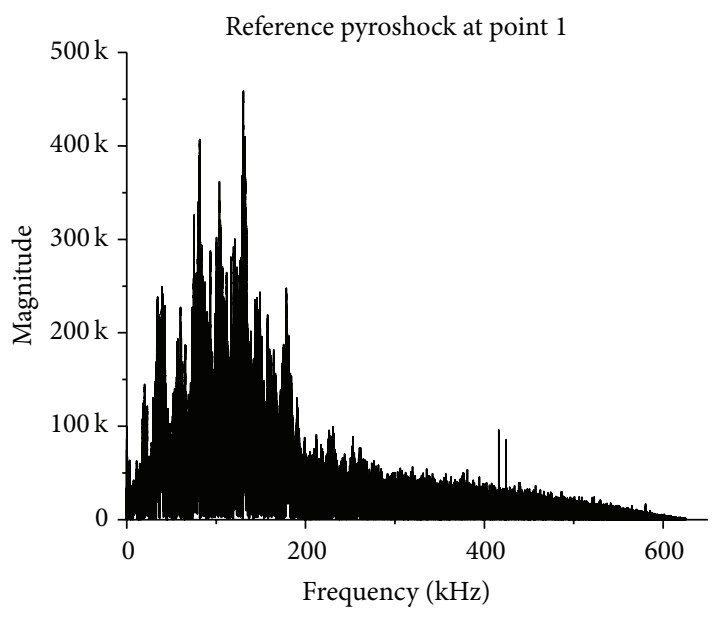

(b)

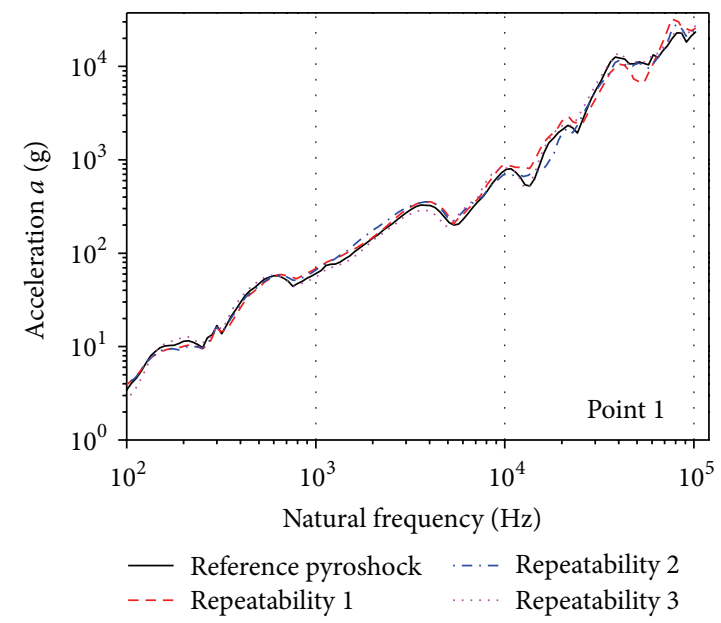

(c)

FIGURE 4: Reference pyroshock signal at Pt1: (a) time-domain, (b) frequency-domain, and (c) SRS response of the reference pyroshock signal and the SRS repeatability.

signals are determined, a reconstructed pyroshock signal can be determined as follows:

$$
\begin{gathered}
P_{\text {recon. }}(k)=\sum_{i=1}^{M} \sum_{j=1}^{N} G_{i, j}(k) L_{i, j}(k), \\
G_{i, j}(k)=\frac{\widetilde{P}_{i, j}(k)}{\widetilde{L}_{i, j}(k)} .
\end{gathered}
$$

Equation (2) shows that the reconstructed pyroshock signal can be obtained by multiplying a gain $G_{i, j}(k)$ by the decomposed laser shock signal $L_{i, j}(k)$, respectively, and the gain $G_{i, j}(k)$ is the ratio of $\widetilde{P}_{i, j}(k)$ to $\widetilde{L}_{i, j}(k)$.

The code for this algorithm was developed in a LabVIEW platform.

\section{Experimental Setups}

In this section, two experimental setups for pyroshock measurement and laser shock measurement from a specimen are first introduced. The specimen used in these experiments was a $2 \mathrm{~mm}$ thick aluminum plate ( $\mathrm{Al} \mathrm{1050)}$ and Figure 2(a) presents the schematic of the aluminum plate specimen with a size of $1700 \times 2500 \times 2 \mathrm{~mm}^{3}$. Then, the settings and operation of the signal processing algorithm are presented too.

3.1. Pointwise Explosive Pyroshock Measurement. For the pyroshock measurement, an initiator as the pointwise explosive was used as a pyroshock source as shown in the inset of Figure 2(a). Figure 2(b) illustrates an initiator mount which was made of a stainless steel material. The initiator mount was used to mount the initiator and clapped at the left end of the specimen plate, as illustrated in Figure 2(a). Once the initiator was detonated, a pyroshock wave was generated and the wave propagated away from the source into the aluminum plate. In this paper, four reference pyroshock signals were considered for the implementation of the signal processing algorithm. Hence, four LDVs (OFV-5000, Polytech) were set up at approximately $3 \mathrm{~m}$ stand-off distance, as shown in Figure 2(c), to simultaneously measure four pyroshock 


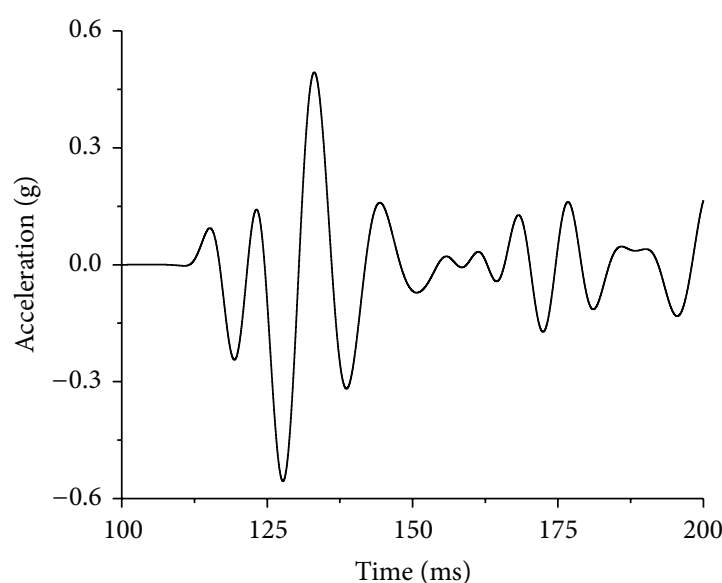

Pyroshock at point 1

_ Low pass $100 \mathrm{~Hz}$

(a)

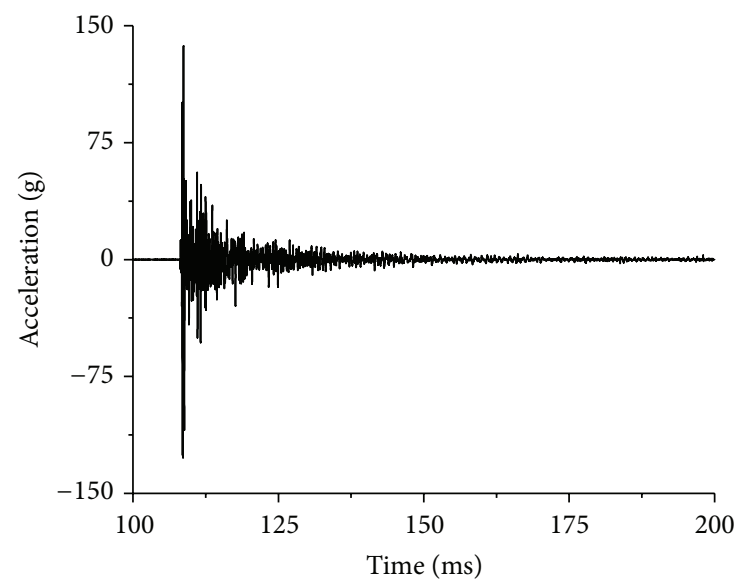

Pyroshock at point 1

— Bandpass ( $1 \mathrm{kHz}-10 \mathrm{kHz})$

(c)

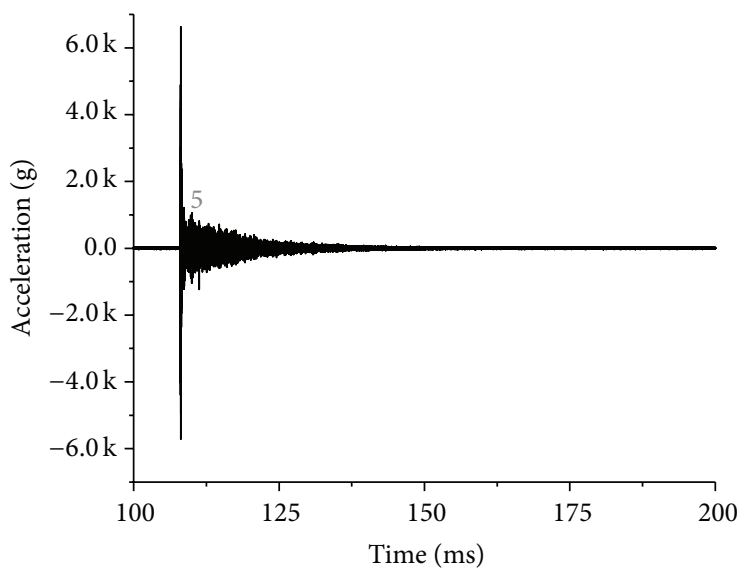

Pyroshock at point 1

High pass $(100 \mathrm{kHz})$

(e)

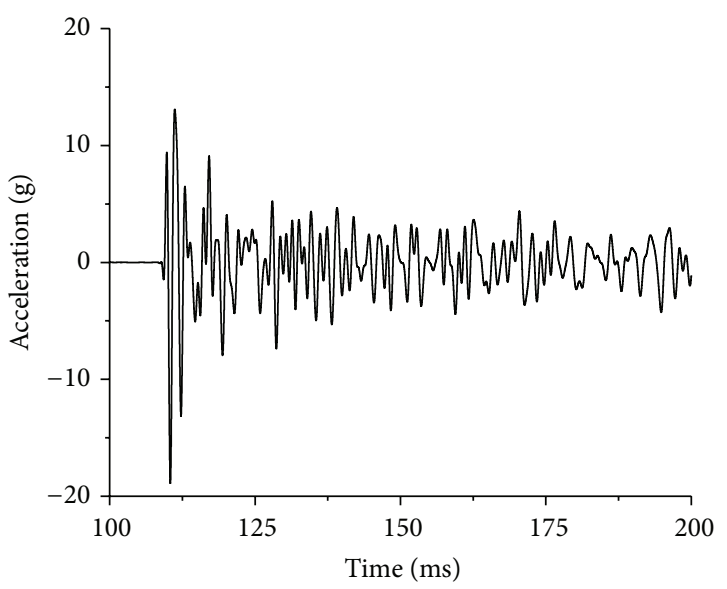

Pyroshock at point 1

- Bandpass $(100 \mathrm{~Hz}-1 \mathrm{kHz})$

(b)

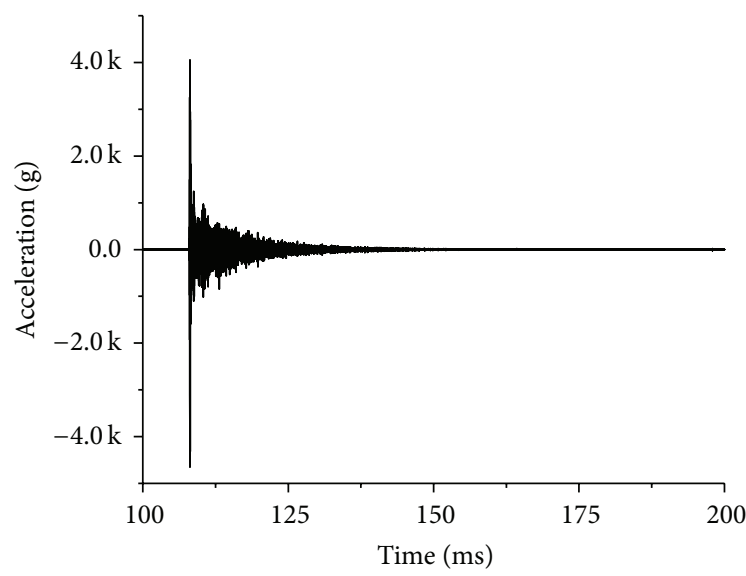

Pyroshock at point 1

- Bandpass (10 kHz-100 kHz)

(d)

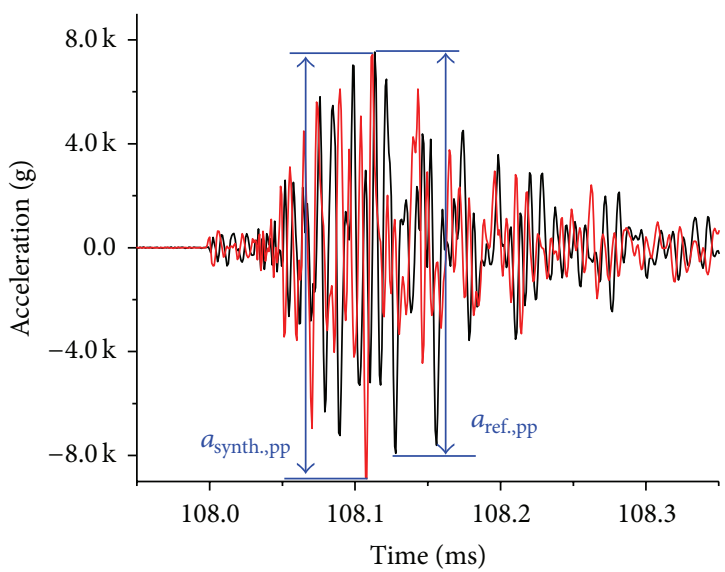

Directly synthesized pyroshock at point 1 Pyroshock at point 1

(f)

Figure 5: Decomposition of the reference pyroshock signal at Ptl with the bandwidths of (a) 0 to $100 \mathrm{~Hz},(\mathrm{~b}) 100 \mathrm{~Hz}$ to $1 \mathrm{kHz},(\mathrm{c}) 1 \mathrm{kHz}$ to $10 \mathrm{kHz}$, (d) $10 \mathrm{kHz}$ to $100 \mathrm{kHz}$, and (e) $100 \mathrm{kHz}$ to $625 \mathrm{kHz}$ and (f) direct synthesizing of the decomposed signals $\left(a_{\text {ref.,pp }}\right.$ and $a_{\text {synth.,pp }}$ are the peak-to-peak accelerations of pyroshock and direct synthesized pyroshock at point 1). 


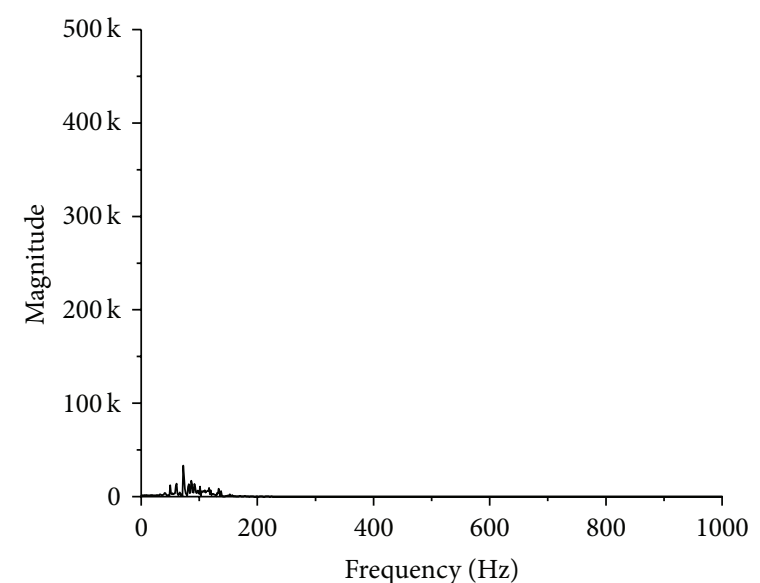

Pyroshock at point 1

— Low pass $100 \mathrm{~Hz}$

(a)

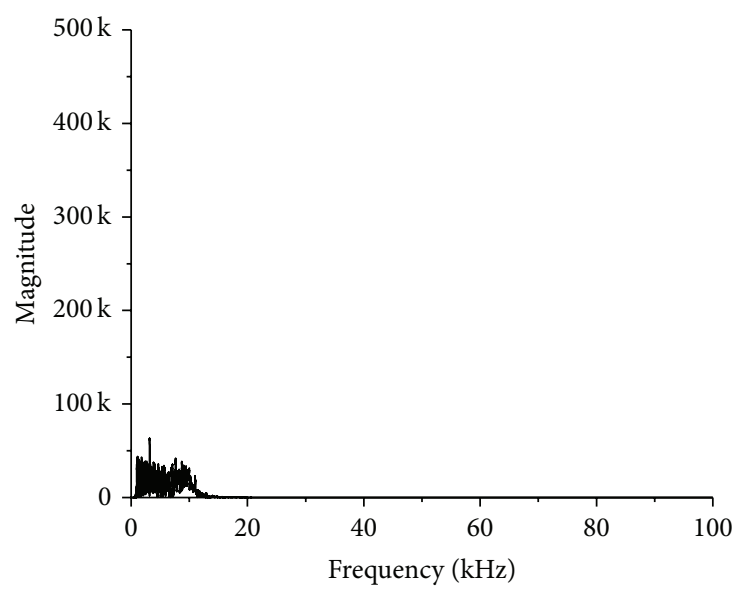

Pyroshock at point 1

— Bandpass (1 kHz-10 kHz)

(c)

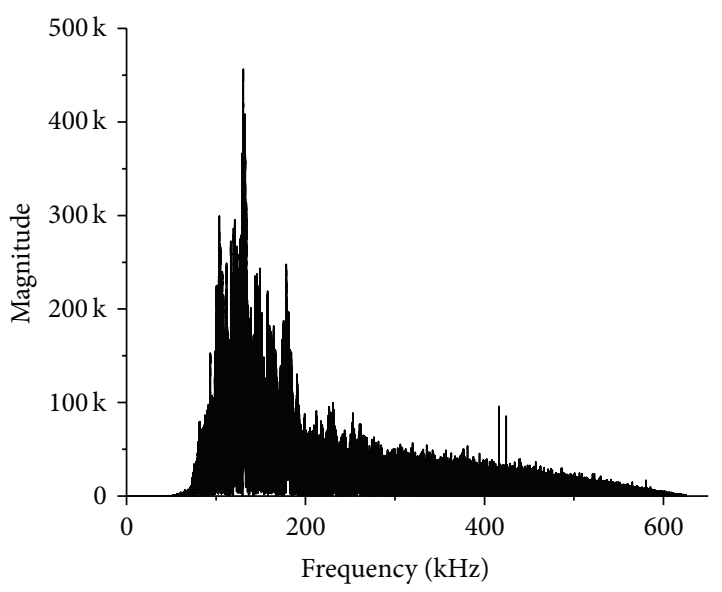

Pyroshock at point 1

— High pass $(100 \mathrm{kHz})$

(e)

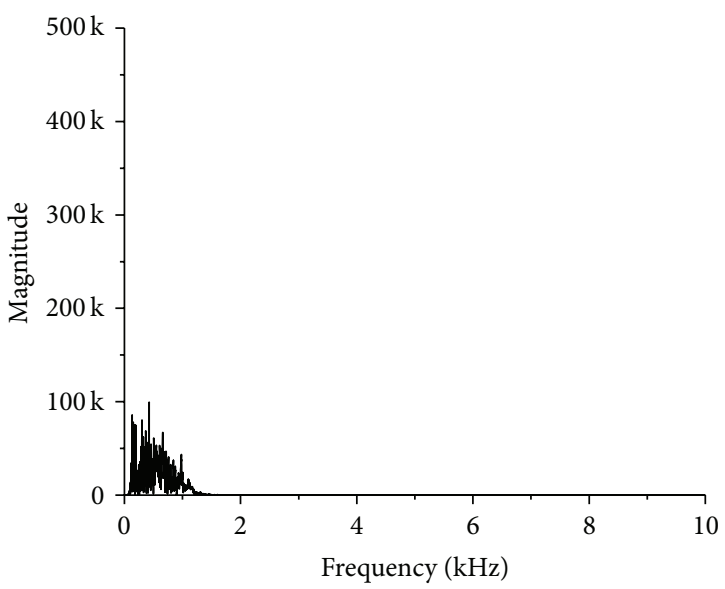

Pyroshock at point 1

— Bandpass $(100 \mathrm{~Hz}-1 \mathrm{kHz})$

(b)

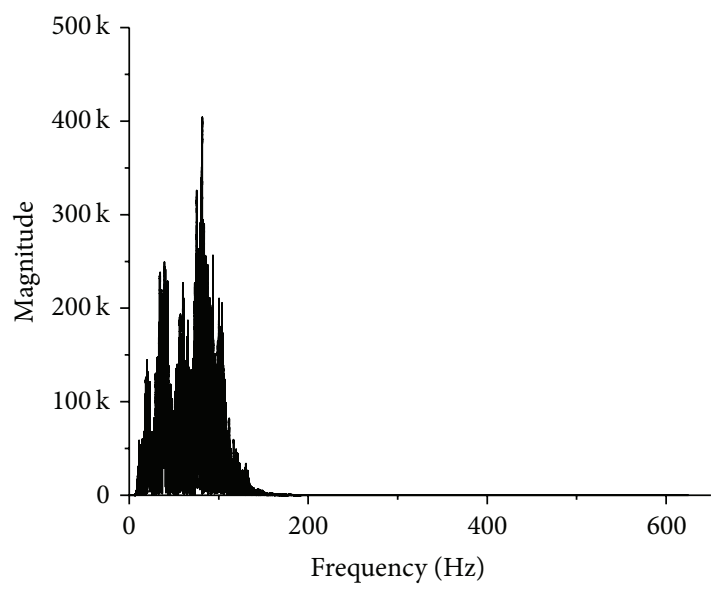

Pyroshock at point 1

— Bandpass (10kHz-100 kHz)

(d)

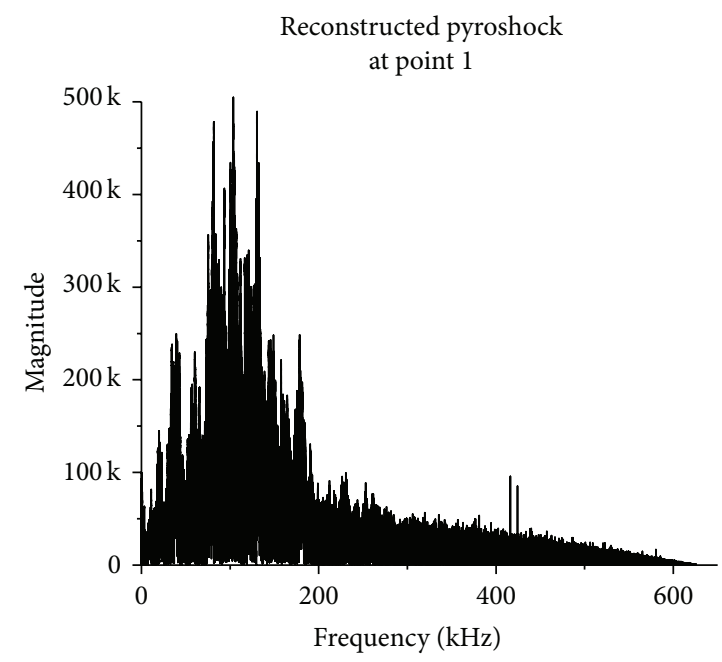

(f)

FiguRE 6: Frequency components of the reference pyroshock signal at Pt1 with respect to the bandwidths of (a) 0 to $100 \mathrm{~Hz}$, (b) $100 \mathrm{~Hz}$ to $1 \mathrm{kHz}$, (c) $1 \mathrm{kHz}$ to $10 \mathrm{kHz}$, (d) $10 \mathrm{kHz}$ to $100 \mathrm{kHz}$, and (e) $100 \mathrm{kHz}$ to $625 \mathrm{kHz}$. (f) Frequency components of the reconstructed pyroshock signal at Pt1. 

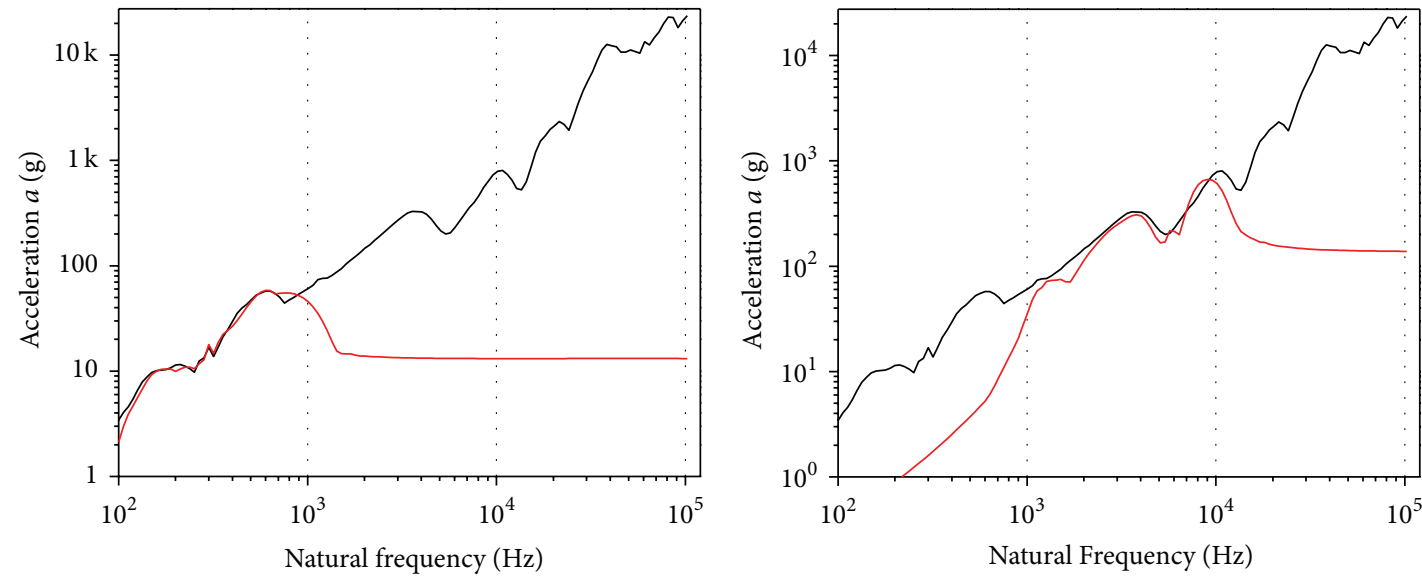

Pyroshock at point 1

- Reference

— Bandpass $(100 \mathrm{~Hz}-1 \mathrm{kHz})$

(a)

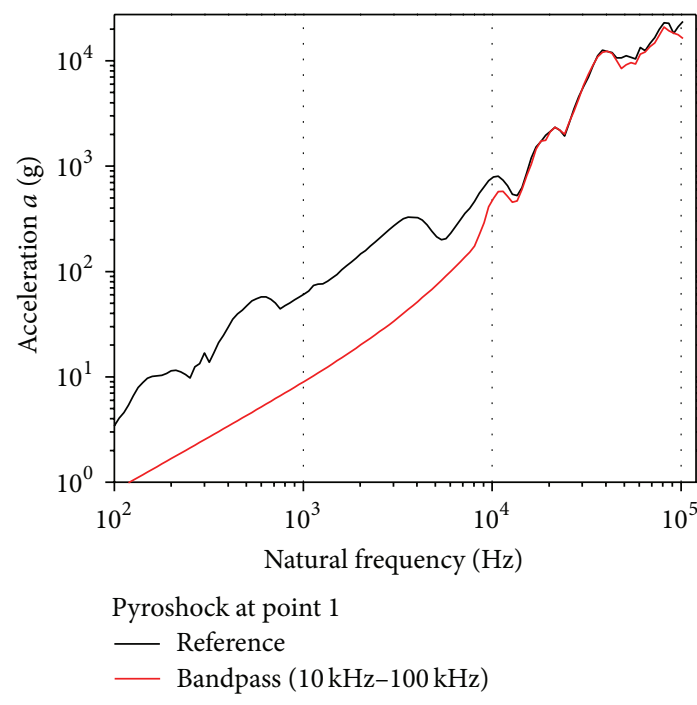

(c)
Pyroshock at point 1

- Reference

— Bandpass (1 kHz-10 kHz)

(b)

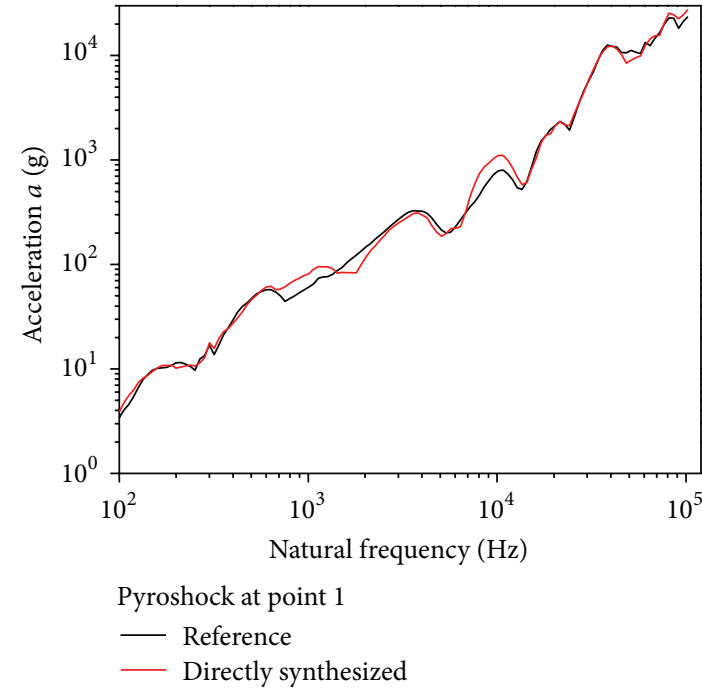

(d)

FIGURE 7: Comparison between the reference SRS curve and the pyroshock SRS curve decomposed in the frequency band of (a) $100 \mathrm{~Hz}$ to $1 \mathrm{kHz}$, (b) $1 \mathrm{kHz}$ to $10 \mathrm{kHz}$, and (c) $10 \mathrm{kHz}$ to $100 \mathrm{kHz}$ and (d) comparison between the reference SRS curve and the SRS curve superposed with the decomposed three pyroshock signals.

TABLE 3: Mean acceleration difference (\%) in SRS between the reconstructed pyroshock signals and reference pyroshock signals.

\begin{tabular}{|c|c|c|c|c|c|c|}
\hline \multirow{2}{*}{$\begin{array}{l}\text { Level } 1(\mathrm{~L} 1) \\
M \text { bandpass filters }\end{array}$} & \multirow{2}{*}{$\begin{array}{l}\text { Level } 2 \text { (L2) } \\
N \text { bandpass filters }\end{array}$} & \multicolumn{4}{|c|}{ Mean acceleration difference (\%) } & \multirow[b]{2}{*}{$\begin{array}{l}\text { Average } \\
\text { Ptl PT4 }\end{array}$} \\
\hline & & Pt1 & $\mathrm{Pt} 2$ & Pt3 & Pt4 & \\
\hline 1 & 1 & 533.7 & 458.9 & 477.4 & 418.6 & 472.15 \\
\hline 3 & 1 & 121.5 & 161.3 & 172.7 & 120.1 & 143.90 \\
\hline 3 & 5 & 35.2 & 60.5 & 82.1 & 37.4 & 53.80 \\
\hline 3 & 10 & 24.5 & 24.2 & 23.6 & 25.9 & 24.55 \\
\hline 3 & 20 & 24.5 & 21.0 & 17.6 & 19.6 & 20.68 \\
\hline 3 & 23 & 23.6 & 19.1 & 18.8 & 17.9 & 19.85 \\
\hline 3 & 25 & 25.0 & 21.4 & 20.6 & 16.9 & 20.98 \\
\hline 3 & 30 & 25.5 & 20.6 & 18.6 & 17.1 & 20.45 \\
\hline 3 & 40 & 28.0 & 22.0 & 20.2 & 23.9 & 23.53 \\
\hline
\end{tabular}




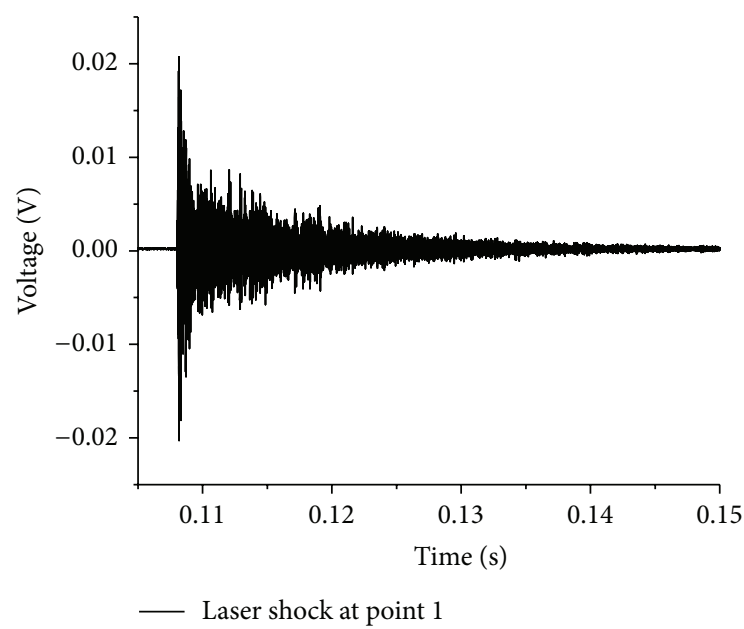

(a)

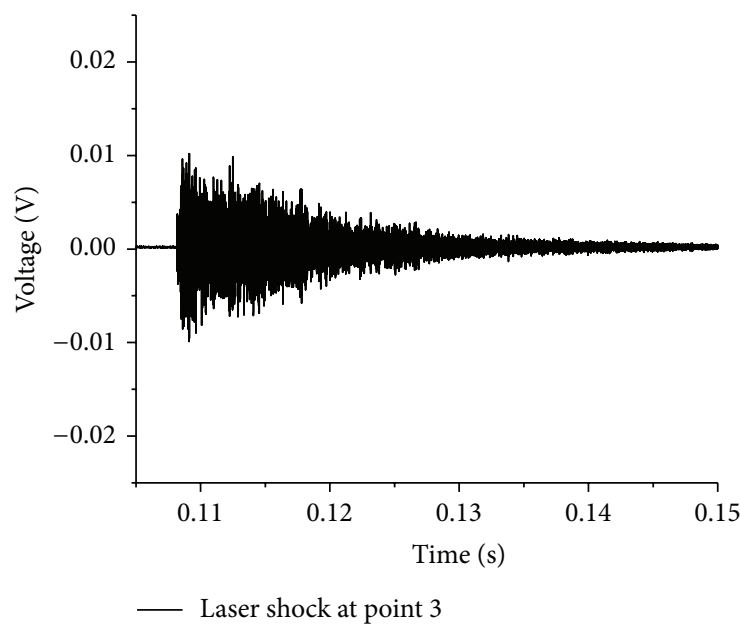

(c)

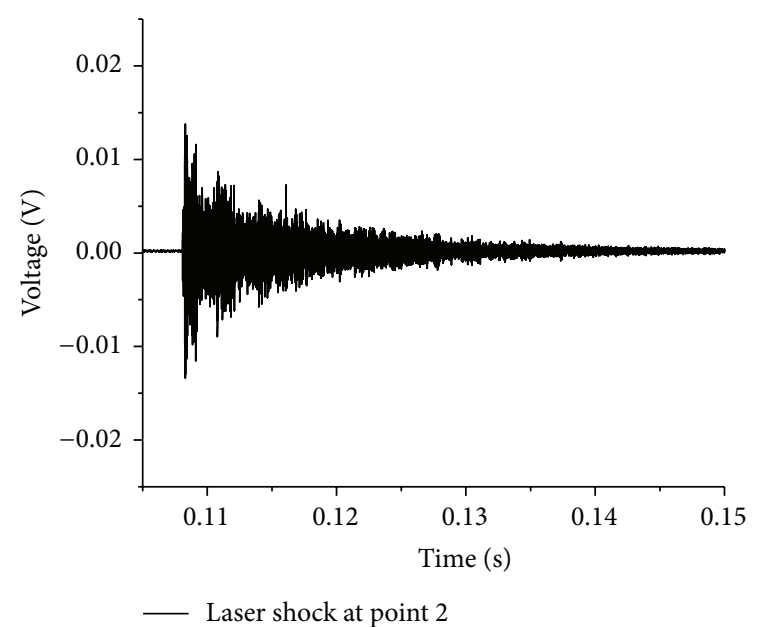

(b)

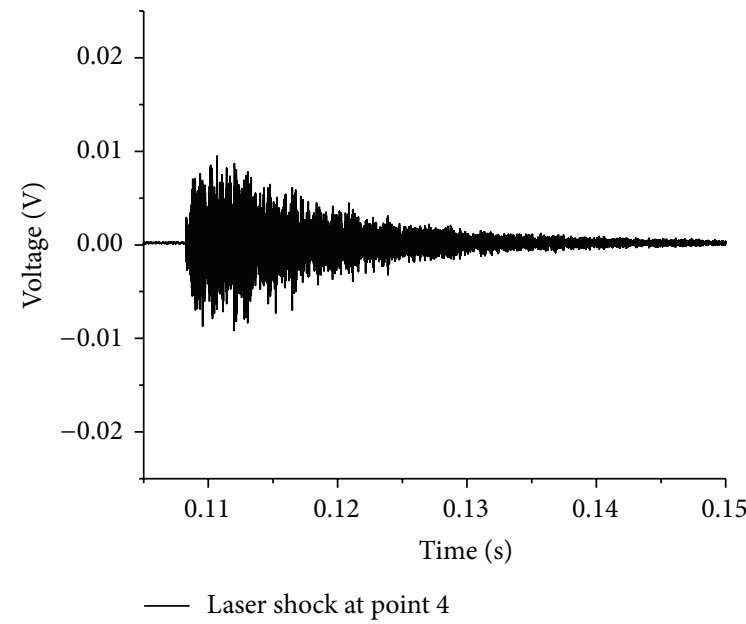

(d)

Figure 8: Laser shock signals at (a) Pt1, (b) Pt2, (c) Pt3, and (d) Pt4.

signals. As shown in Figure 2(a), the four sensing points (Pt1-Pt4) were located along a line with the distances of $250 \mathrm{~mm}, 500 \mathrm{~mm}, 1000 \mathrm{~mm}$, and $1500 \mathrm{~mm}$ from the initiator mount. During the measurement, retroreflective films were used to minimize the speckle noise to improve the signalto-noise ratio (SNR) of the sensing signal. Thus, they were attached on the local sensing points (Pt1-Pt4), respectively. The band of interest for the pyroshock signal in this paper was $100 \mathrm{~Hz}$ to $100 \mathrm{kHz}$. Therefore, the four pyroshock signals were measured with a low pass of $100 \mathrm{kHz}$ in the respective controllers of the LDVs. Afterwards, the signals were acquired and digitized through the four channels of an 8-channel highspeed digitizer (NI PCI-5105) at a sampling time interval of $T=0.8 \mu$ s and a signal length of $k=900,000$ samples in a LabVIEW platform. In the measurement, high sampling frequency was selected in order to preserve the acquired pyroshock signal without aliasing, as well as the waveform shape for time-domain analysis. After the acquisition, four pyroshock signals were stored in a computer for later implementation of the signal processing algorithm.
3.2. Laser Shock Measurement. Figure 3 demonstrates the experimental setup for the laser shock measurement on the aluminum plate used in the pyroshock measurement. As illustrated in Figure 3, the experimental setup was configured with a Q-switched Nd:YAG diode pumped solid state laser (QL) with $1064 \mathrm{~nm}$ wavelength, a QL controller, a galvanometric laser mirror scanner (LMS), a laser shock sensor system, and a computer with a LabVIEW platform for data acquisition and synchronization control.

The QL was used as a laser shock source and controlled by the QL controller. The LMS has two mirrors with $1064 \mathrm{~nm}$ reflective coating, where each mirror is mounted on a galvanometer. Hence, the LMS was used to maneuver the laser pulse to the sensing point. In the laser shock sensor system, an in-line bandpass filter was used during the laser shock signal acquisition. Then, an omnidirectional amplifierintegrated accelerometer (PZT transducer) was selected for the system to measure a laser shock signal. It has broadband frequency characteristics with the cutoff frequency of $2 \mathrm{MHz}$ and the sensitivity of up to $316 \mathrm{~V} / \mathrm{mm} / \mathrm{s}$. In regard to its high 


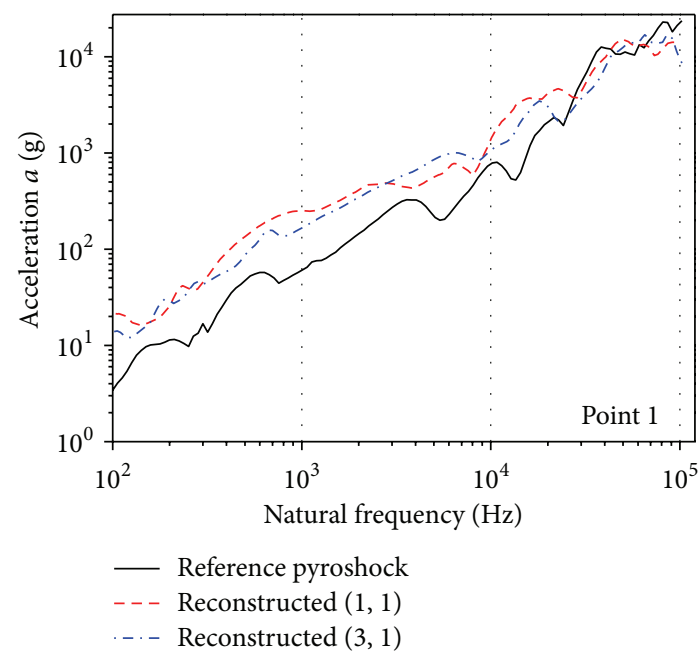

(a)

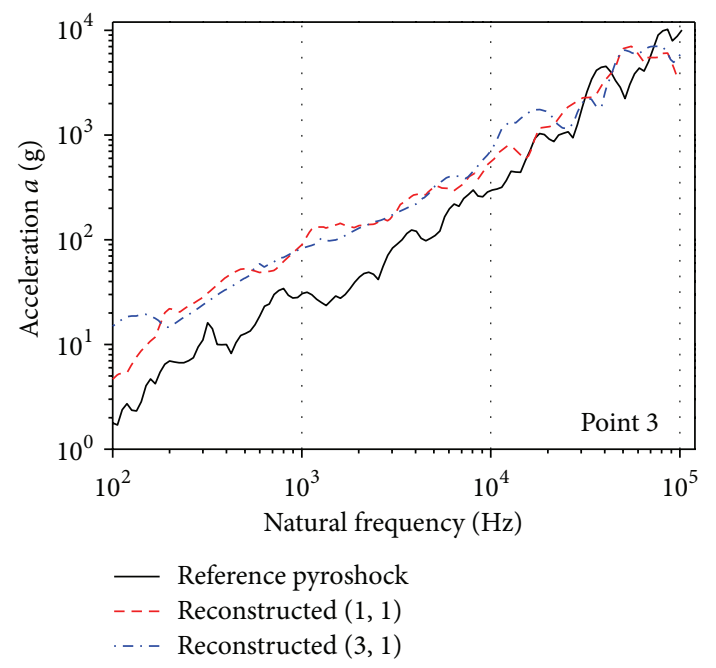

(c)

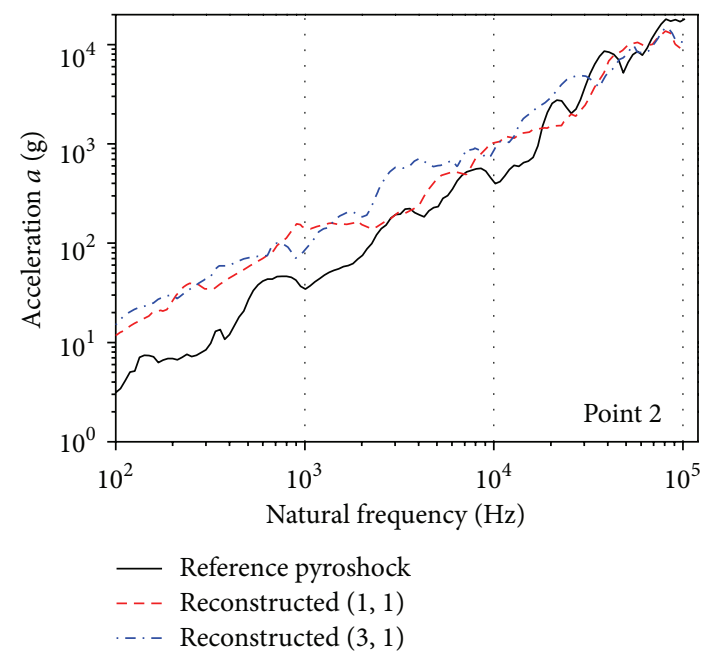

(b)

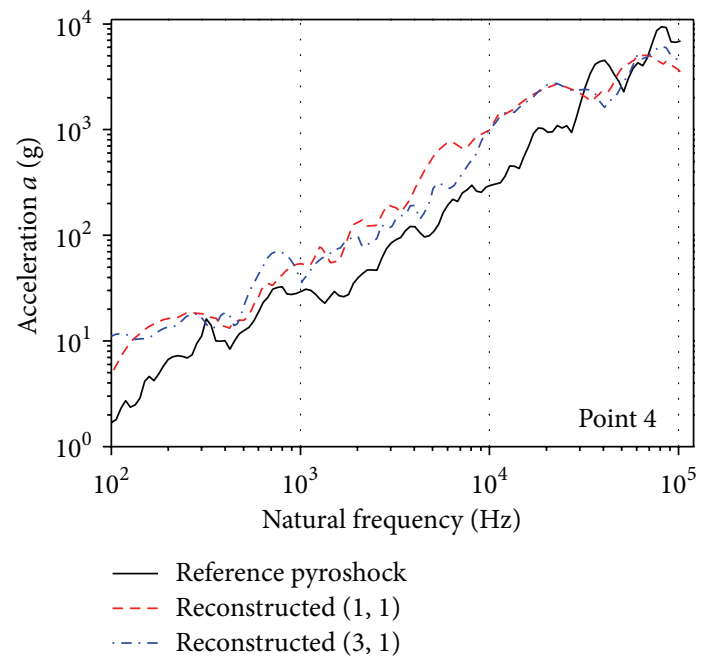

(d)

Figure 9: SRS curve comparison between the reference and reconstructed pyroshock signals at (a) Ptl, (b) Pt2, (c) Pt3, and (d) Pt4 in the decomposition cases of $(1,1)$ and $(3,1)$.

sensitivity in comparison to $\operatorname{LDV}(0.2 \mathrm{~V} / \mathrm{mm} / \mathrm{s})$, the sensor was more suitable to be used in the laser shock measurement. As shown in the inset of Figure 3, the accelerometer was bonded on the surface of the initiator mount (Figure 2(b)) using a cyanoacrylate adhesive. Lastly, a computer was used to synchronize the laser shock generation and measurement events and stored the laser shock signal for later processing.

The LMS maneuvered the laser pulse set at laser energy of $2.5 \mathrm{~mJ}$ towards the sensing point as shown in Figure 3. The four laser shock signals were acquired one point by one point. When the laser pulse impinged on the surface of the specimen, the laser shock was generated based on a thermoelastic regime. Then, the laser shock wave propagated away from the impingement point to the accelerometer. Using the reciprocity of wave propagation time, the laser shock wave was regarded as a wave generated at the excitation point and received from the laser impingement point. After that, the laser shock signal was filtered in the in-line bandpass filter with the cuton of $100 \mathrm{~Hz}$ and cutoff of $100 \mathrm{kHz}$ simply because the frequency band to be analyzed in terms of SRS was the frequency band of $100 \mathrm{~Hz}$ to $100 \mathrm{kHz}$. Subsequently, the signal was acquired into the high-speed digitizer (NI PCI-5105) at the same sampling time interval and signal length as in the pyroshock measurement, $T=0.8 \mu \mathrm{s}, k=900,000$ samples in the LabVIEW platform.

3.3. Signal Processing Algorithm Setting and Operation. After the pyroshock and laser shock measurements, the pyroshock and laser shock signals were stored into the computer. Before these signals were processed by the proposed algorithm, the two settings of the algorithm were considered and set, as shown in Table 1.

One bandpass filter $(M=N=1)$ was chosen in L1 and L2, respectively. Both high and low cutoff frequencies were set to $100 \mathrm{~Hz}$ to $100 \mathrm{kHz}$. In the second case, three bandpass 


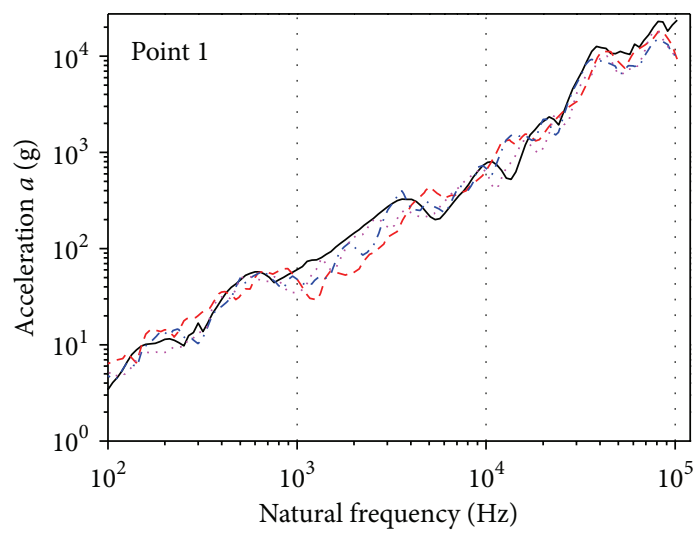

$\begin{array}{lll}\text { - Reference pyroshock } & \cdots & \text { Reconstructed }(3,10) \\ \text { - - - Reconstructed }(3,5) & \cdots & \text { Minimized and reconstructed }(3,23)\end{array}$

(a)

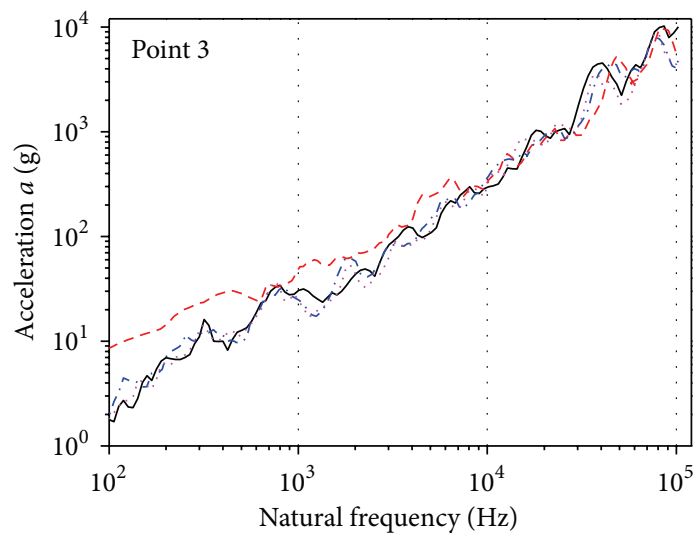

$\begin{array}{lll}\text { — Reference pyroshock } & - & \text { Reconstructed }(3,10) \\ \text { - - Reconstructed }(3,5) & \ldots . . & \text { Minimized and reconstructed }(3,23)\end{array}$

(c)

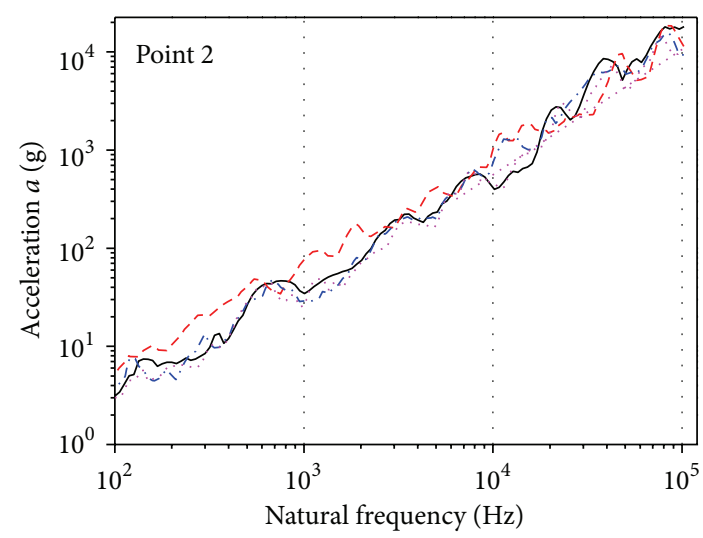

$\begin{array}{lll}\text { —- Reference pyroshock } & \ldots & \text { Reconstructed }(3,10) \\ \text { - - Reconstructed }(3,5) & \cdots & \text { Minimized and reconstructed }(3,23)\end{array}$

(b)

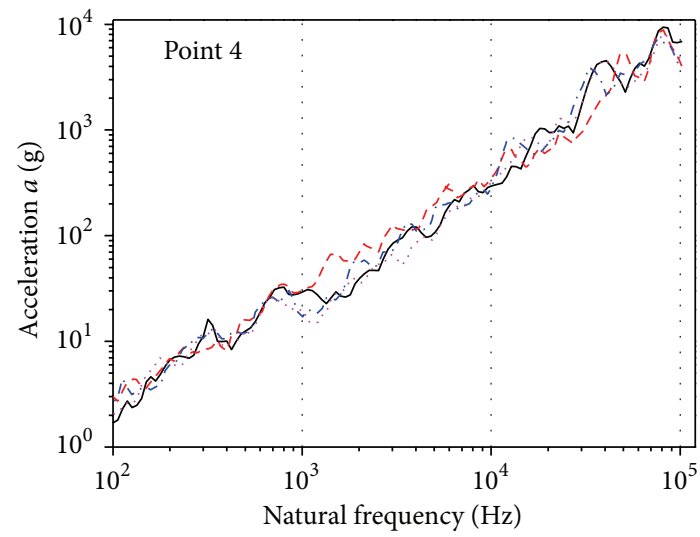

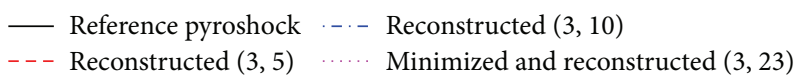

(d)

FIGURE 10: SRS curve comparison between the reference pyroshock signal and the pyroshock signals reconstructed with hieratical subband decomposition and MAD minimization at (a) Pt1, (b) Pt2, (c) Pt3, and (d) Pt4 in cases of three different $N$ bandpass filters in Level 2 ((3, $N)$ cases).

filters ( $M=3, N=1)$ were chosen and the respective high and low cutoff frequencies were set to $100 \mathrm{~Hz}$ to $1 \mathrm{kHz}, 1 \mathrm{kHz}$ to $10 \mathrm{kHz}$, and $10 \mathrm{kHz}$ to $100 \mathrm{kHz}$. This set of high and low cutoff frequencies was chosen because the midfield is between $10 \mathrm{kHz}$ and $100 \mathrm{kHz}$ and the far-field is below $10 \mathrm{kHz}$ [13] and also the frequency scale of an SRS curve is plotted on the 3cycle log-scale $x$-axis in a log-log graph. As shown in Table 1 , four different $N$ bandpass filters in L2 were chosen. Each decomposed signal after L1 was decomposed again with the $\mathrm{N}$ bandpass filters with equal bandwidths, respectively. First, one bandpass filter was set in L2 for processing, and then the processing is followed by 5,10 , and 20 bandpass filters in the second case.

After the parameter setting, the reference pyroshock signal and the laser signal, which were measured at Ptl, were inputted into the subbands decomposition (Figure 1). After the decomposition, $P_{M, N}(k)$ and $L_{M, N}(k)$ were obtained. Then, in the wave matching, the enveloped signals $\widetilde{P}_{M, N}(k)$ and $\widetilde{L}_{M, N}(k)$ were extracted from the decomposed signals. Subsequently, the gain $G_{M, N}(k)$ based on (3) was determined, and the synthesized pyroshock signal at Ptl could be determined through (2). After the processing of Ptl, the processing was repeated to obtain the synthesized pyroshock signals at $\mathrm{Pt} 2, \mathrm{Pt} 3$, and Pt4, respectively. Also, after the processing in case 1 was completed, the processing was repeated for case 2 with the same manner, where four synthesized pyroshock signals were generated with respect to $N=1 \mathrm{in} \mathrm{L2}$, and then followed by 5,10 , and 20 .

After all these synthesized pyroshock signals were obtained, the similarity between the SRS curves of the synthesized pyroshock signal and the reference pyroshock signal was evaluated based on the mean acceleration difference (MAD, \%) as follows:

$$
\operatorname{MAD}(\%)=\left(\frac{1}{Z} \sum_{i=0}^{Z-1}\left|\frac{a_{P}-a_{\text {recon. }}}{a_{P}}\right|\right) \times 100 \%
$$


where $Z$ is the total number of acceleration values in the SRS curve, $a_{P}$ is the acceleration in the SRS curve of a reference pyroshock signal, and $a_{\text {recon. }}$ is the acceleration of the SRS curve for a reconstructed pyroshock signal.

\section{Results and Discussion}

4.1. Pyroshock Characteristics Study. Before the implementation of the proposed signal processing algorithm, the characteristics of pyroshock signals were investigated. As mentioned in Section 2, for the algorithm to construct a pyroshock signal based on a laser shock signal, the assumption that the pyroshock signal is governed by the principle of wave superposition was also verified. For that, the reference pyroshock signal at Ptl was used to verify the agreement of the superposition principle.

Figure 4(a) shows the reference pyroshock signal plotted in the time domain. The peak-to-peak amplitude in acceleration of the pyroshock $a_{\text {ref.,pp }}$ was $16,322.5 \mathrm{~g}$. Figure $4(\mathrm{~b})$ shows the frequency components of the pyroshock obtained by the fast Fourier transform (FFT) with a frequency range of up to $625 \mathrm{kHz}$. The area under the curve is equal to the energy of the signal. As shown in Figure 4(b), the energy of the pyroshock was concentrated below the frequency of $130.3 \mathrm{kHz}$. In addition, the frequencies of $81.5 \mathrm{kHz}, 103.7 \mathrm{kHz}$, and $130.3 \mathrm{kHz}$ had higher energy among the other frequency components. Figure 4(c) shows the SRS response of the pyroshock signal from $100 \mathrm{~Hz}$ to $100 \mathrm{kHz}$. The corresponding acceleration range with respect to the natural frequency was approximately from $3.8 \mathrm{~g}$ to $27,676.4 \mathrm{~g}$. Then, Figure 4 (c) also includes the SRS curves with respect to the other three repeated pyroshock measurements. As summarized in Table 2, the average of the MADs over the four points calculated using (4) in the pointwise explosive tests was $19.6 \%$.

To verify if the pyroshock signal is governed by the principle of superposition, the pyroshock signal at Pt1 was first decomposed into five different subbands with the bandwidths of 0 to $100 \mathrm{~Hz}, 100 \mathrm{~Hz}$ to $1 \mathrm{kHz}, 1 \mathrm{kHz}$ to $10 \mathrm{kHz}, 10 \mathrm{kHz}$ to $100 \mathrm{kHz}$, and $100 \mathrm{kHz}$ to $625 \mathrm{kHz}$, respectively. Figures 5(a)5 (e) show the decomposed signals in the five subbands. The subbands with higher frequencies included the decomposed pyroshock signals with higher amplitudes. Even if the reference pyroshock signal was acquired with the $100 \mathrm{kHz}$ lowpass filter built in the LDV, the amplitude of the decomposed signal with the $100 \mathrm{kHz}$ numerical high-pass filter, as illustrated in Figure 5(e), had higher peak-to-peak acceleration in comparison to the other decomposed signals. To reconstruct the pyroshock signal, all of the decomposed pyroshock signals were just simply summed up, and the reconstructed pyroshock signal was obtained as shown in Figure 5(f). As shown in Figure 5(f), the reconstructed pyroshock signal exhibited the peak-to-peak acceleration $a_{\text {recon.,pp }}$ obtained at $15,446.55 \mathrm{~g}$, and it has only $5.4 \%$ difference from the peak-topeak acceleration, $a_{\text {ref.,pp }}$, of the reference pyroshock signal at Pt1. The difference was suspected due to some data distorted during the signal decomposition and synthesis processes.
Next, the frequency components of the decomposed signals were obtained by using FFT as shown in Figures 6(a)6(e), respectively. The energy of the decomposed pyroshock signals increased as the corresponding frequency bandwidth increased. Within them, the frequency components from $10 \mathrm{kHz}$ to $625 \mathrm{kHz}$ possessed the most energy in the reference pyroshock signal. In addition, the frequencies of $81.5 \mathrm{kHz}$, $103.7 \mathrm{kHz}$, and $130.3 \mathrm{kHz}$ had higher energy among the other frequency components. This has reflected that the peak-topeak acceleration of the signal for Figure 5(e) stood at the highest amplitude value. Then, the shape of the frequency components for the reconstructed pyroshock signal was also similar to the reference pyroshock signal at Ptl as shown in Figure 6(f).

Figures 7(a)-7(c) demonstrate the SRS curves between $100 \mathrm{~Hz}$ and $100 \mathrm{kHz}$ of the decomposed pyroshock signals, respectively. Frequency range is important because it is considered as the far- and midfield frequency components in the SRS analysis of the pyroshock. As shown in Figure 7(a), the SRS curve of the pyroshock signal decomposed from $100 \mathrm{~Hz}$ to $1 \mathrm{kHz}$ showed a good match to that of the reference pyroshock signal at Pt1 in the frequency range of $100 \mathrm{~Hz}$ to $1 \mathrm{kHz}$. Then, as shown in Figures 7(b) and 7(c), the respective SRS curves of the decomposed pyroshock signals also showed good agreement with those of the reference pyroshock signals in the respective decomposed frequency zones. As presented in Figure 7(d), the SRS curve of the pyroshock signal superposed with the decomposed three time-domain signals also showed good agreement with the SRS curve of the reference pyroshock signal at Ptl. Consequently, as proven in Figure $7(\mathrm{~d})$, the pyroshock signal can be analyzed with the wave superposition principle.

4.2. Pyroshock SRS Generation Using a Laser Shock. With the validated assumption, the signal processing algorithm proposed to reconstruct a pyroshock signal using a laser shock signal was verified in this section. The generation mechanisms of the pyroshock and laser shock are different from each other; the origin of both resulting waves is commonly stress waves. On the other hand, their frequency components and amplitude are different.

Figures 8(a)-8(d) show the laser shock signals at Pt1, Pt2, Pt3, and Pt4, respectively. Each laser shock signal was measured at the submillivolt level. Each laser shock signal was inputted into the laser shock-based pyroshock reconstruction algorithm presented in Section 2, together with the reference pyroshock signal at the corresponding measurement point. Both signals were decomposed in the way listed in Table 1 and their SRSs were matched and synthesized. Moreover, the same processing was performed for the other three points.

Figures 9 and 10 show the SRS curves at Pt1, Pt2, Pt3, and Pt4, respectively, which were generated by the laser shock-based pyroshock SRS reconstruction algorithm. Table 3 summarizes the MAD of the SRS curves between the reconstructed pyroshock signals and the reference pyroshock signals, which was calculated using (4).

For the single band and nonhieratical decomposition cases, $(M, N)=(1,1)$ and $(1,3)$, the SRS curves of both 
simulated pyroshock signals showed very low similarity to the reference pyroshock signals as shown in Figure 9. The MAD was also very high as presented in Table 3 . For the cases using the hieratical method and more bands, $(3,5)$ and $(3,10)$, the MADs in the SRS curves of the reconstructed pyroshock signals were improved and demonstrated better similarity as the $N$ increased from 5 to 10 subbands. Therefore, the simple bisection method was used to find $N$ minimizing the average MAD over the four points in SRSs between the reconstructed and reference pyroshocks, where the boundary values for $N$ were 20 and 40 . The iteration converged into $N=23$ and the average of the resulted MADs over the four points was $19.85 \%$, which is an acceptable value for the prediction of the SRSs of real pyroshocks because the ballistic pyroshock measurement experiments using the actual explosives showed only $19.6 \%$ in the average of MAD as reported in Section 4.1.

Table 3 indicates that some cases show better results at point 3 or point 4 . These results have shown that the similarity of the reconstructed SRS is dependent on the number of bandpass filters set. A pyroshock signal was decomposed by a number of bandpass filters to allow the respective decomposed laser signals to match with the decomposed pyroshock signals. However, during the filtering process, some of the dominant frequency components of the decomposed pyroshock signals, which have the dominant energy to reconstruct an SRS curve, might be filtered out from the passband of the bandpass filters. Then, with both decomposed signals, the SRS matching synthesis process, as shown in Figure 1, could not accurately reconstruct the acceleration amplitude of the pyroshock and eventually caused the low similarity between the acceleration amplitudes of the reconstructed SRS and the reference SRS.

As mentioned in Section 1, the signal processing algorithm can be further developed to reconstruct a pyroshock signal at any arbitrary point at a target specimen. Thus, the settings of the algorithm have to be statistically considered and acted as a common setting for a pyroshock simulator. Thus, the cases with $M=3$ and $N=23$ were the best settings to be employed in the signal processing algorithm that will be incorporated with the pyroshock simulator for the SRS reconstruction based on $2 \mathrm{~mm}$ aluminum plate at any arbitrary point. As for the signal processing time of the case $(3,23)$, it took $1.75 \mathrm{~min}$ using a 64-bit PC (Intel i7 CPU at $2.67 \mathrm{GHz}$ ) with 4 GByte RAM. These results have proven that the SRS curves of a pointwise explosive pyroshock can be estimated and reconstructed at multiple points in a structure using a laser shock with much lower amplitude and wider frequency range and the proposed laser shock-based pyroshock SRS reconstruction algorithm.

\section{Conclusion}

The laser shock-based pyroshock SRS reconstruction algorithm was proposed for the case of a pointwise explosiveinduced pyroshock. The algorithm was based on the new idea that the generation mechanisms of the pyroshock and laser shock are different but the origin of both resulting waves is commonly the stress wave even if their frequency components and amplitude are different. The SRS is an important tool to analyze and quantify a pyroshock. The near-field pyroshock is avoided in the pyrotechnic design level but the mid- and far-field pyroshocks can easily cause failures in electronic and optical components sensitive to high frequency energy below $100 \mathrm{kHz}$ and failures in relay and magnetic components sensitive to low frequency ranges from $2 \mathrm{kHz}$ to $4 \mathrm{kHz}$. Therefore, in this study, the proposed algorithm was concentrated on the frequency range between $100 \mathrm{~Hz}$ and $100 \mathrm{kHz}$ to reconstruct the pyroshock with the laser shock. As for the experiments, the laser shock was measured using the four-point excitation by the Q-switched laser and the single accelerometer in the aluminum plate and the pyroshock measurement was carried out using the single initiator (pointwise explosive) and four LDVs in the same plate. For signal processing, it was verified that the measured pyroshock waves are governed by the wave superposition principle. The proposed signal processing method consisted of the hieratical subband decomposition and the SRS matching synthesis. Two levels of subband decomposition were chosen and a Butterworth IIR bandpass filter was used for subbands decomposition. Then, in the SRS matching synthesis, all the envelopes of the decomposed signals were extracted and the gain was determined. Finally, the gained decomposed laser shock signals were synthesized to reconstruct the SRS of the pyroshock signal. The same process was done for the different points in the specimen, Pt1-Pt4. The similarities between the SRS curves of the reference and reconstructed pyroshocks were evaluated by using MAD (\%). Through the minimization of the MAD in SRS between the reconstructed and reference pyroshocks, $19.85 \%$ was achieved in terms of the average of the MADs over the four points, which was a satisfiable value because the ballistic pyroshock measurement experiments using the actual explosives showed to be $19.6 \%$ in the average of MADs over the four points. The results showed also that the similarity between the reconstructed and reference SRS curves was dependent on the passband bandwidth being set in the bandpass filters as used in the subband decomposition process. For that, the proposed signal processing algorithm can be improved in the future by including a feature where the passband bandwidth of the bandpass filters can be set based on the dominant frequency energy of a reference pyroshock, so that the signal processing algorithm can reconstruct an SRS curve with the better similarity level. Lastly, since the SRS curves of the pyroshock can be predicted with the proposed laser shock-based pyroshock SRS reconstruction method, expensive, laborious, and timeconsuming pyroshock experiments which are also dangerous to both the structure itself and testers can be minimized in the development of pyrotechnical devices.

\section{Conflict of Interests}

The authors declare that there is no conflict of interests regarding the publication of this paper. 


\section{Acknowledgments}

This research was supported by Space Core Technology Development Program (2013042548) and the Basic Science Research Program (2011-0010489) and through the National Research Foundation of Korea funded by the Ministry of Education, Science and Technology. This study was also supported by the University Collaboration Enhancement Project of the Korea Aerospace Research Institute.

\section{References}

[1] J.-R. Lee, C. C. Chia, and C.-W. Kong, "Review of pyroshock wave measurement and simulation for space systems," Measurement, vol. 45, no. 4, pp. 631-642, 2012.

[2] E. Fillippi, H. Attouoman, and C. Conti, "Pyroshock simulation using the alcatel etca test facility," in Launch Vehicle Vibrations, 1st European Conference, CNES, Toulouse, France, 1999.

[3] M. G. Ryschkewitsch, "Pyroshock test criteria," NASA Technical Standard NASA-STD-7003, NASA, Washington, DC, USA, 2011.

[4] M. Gherlone, D. Lomario, M. Mattone, and R. Ruotolo, "Application of wave propagation to pyroshock analysis," Shock and Vibration, vol. 11, no. 3-4, pp. 145-156, 2004.

[5] J.-R. Lee, H. Jeong, and C.-W. Kong, "Wave propagation visualization in an experimental model for a control rod drive mechanism assembly," Nuclear Engineering and Design, vol. 241, no. 9, pp. 3761-3767, 2011.

[6] M. R. Brake, "An inverse shock response spectrum," Mechanical Systems and Signal Processing, vol. 25, no. 7, pp. 2654-2672, 2011.

[7] M. T. Hale and R. Adhami, "Time-frequency analysis of shock data with application to shock response spectrum waveform synthesis," in Proceedings of the IEEE Southeastcon, vol. 1, pp. 213-217, Williamsburg, VA, USA, April 1991.

[8] V. X. Afonso, W. J. Tompkins, T. Q. Nguyen, and S. Luo, "ECG beat detection using filter banks," IEEE Transactions on Biomedical Engineering, vol. 46, no. 2, pp. 192-202, 1999.

[9] A. N. Akansu and R. A. Haddad, Multiresolution Signal Decomposition: Transforms, Subbandss, and Waveletsd, Academic Press, San Diego, Calif, USA, 2nd edition, 2001.

[10] J. Liu and W. Cao, "Fast algorithms of slight signal decomposition and reconstruction," in Proceedings of the 3rd International Conference on Computational Electromagnetics and its Applications (ICCEA '04), pp. 115-117, November 2004.

[11] L. M. A. Augilar, C. Robledo-Sanchez, M. L. A. Carrasco, and M. M. M. Otero, "The principle of superposition for waves: the amplitude and phase modulation phenomena," Applied Mathematics \& Information Science, vol. 6, no. 2, pp. 307-315, 2012.

[12] S. Choi and Z. Jiang, "Comparison of envelope extraction algorithms for cardiac sound signal segmentation," Expert Systems with Applications, vol. 34, no. 2, pp. 1056-1069, 2008.

[13] D. R. Mulville, "Pyroshock test criteria," NASA Technical Standard NASA-STD-7003, NASA, Washington, DC, USA, 1999. 

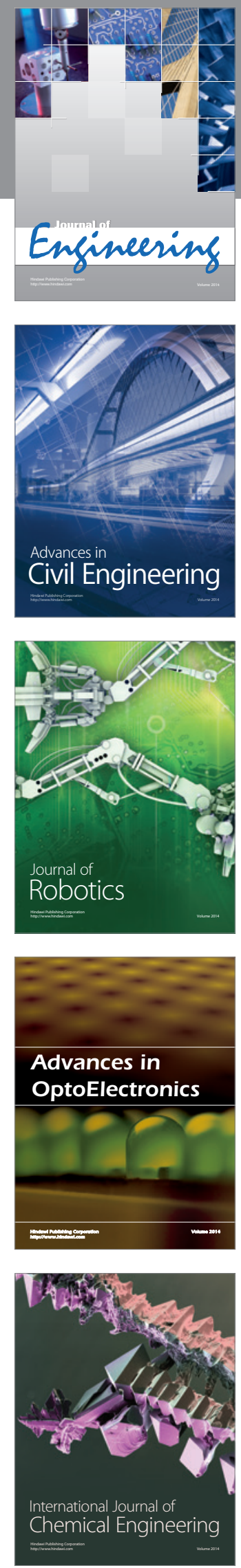

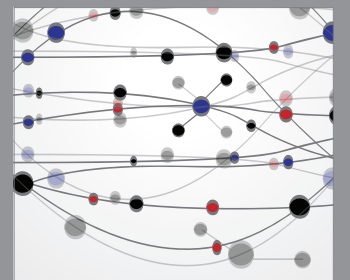

The Scientific World Journal
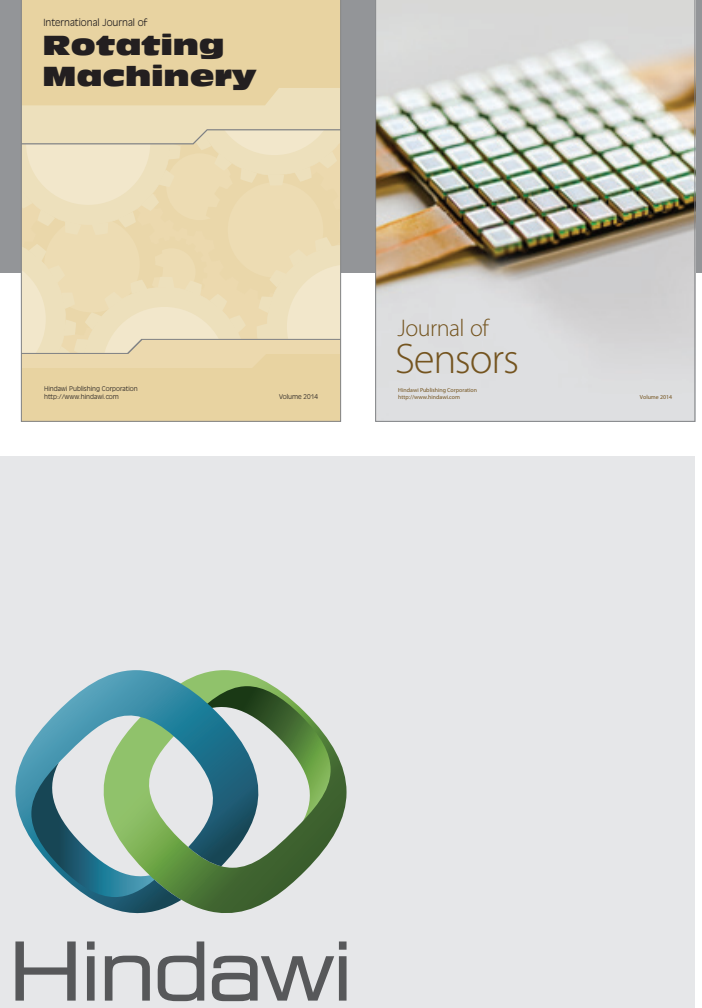

Submit your manuscripts at http://www.hindawi.com
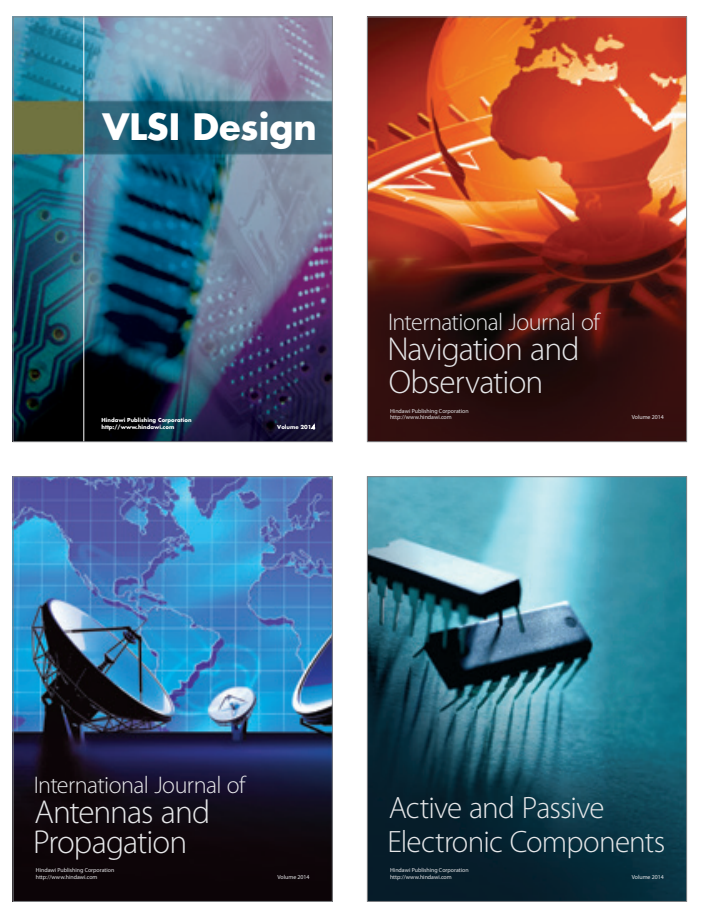
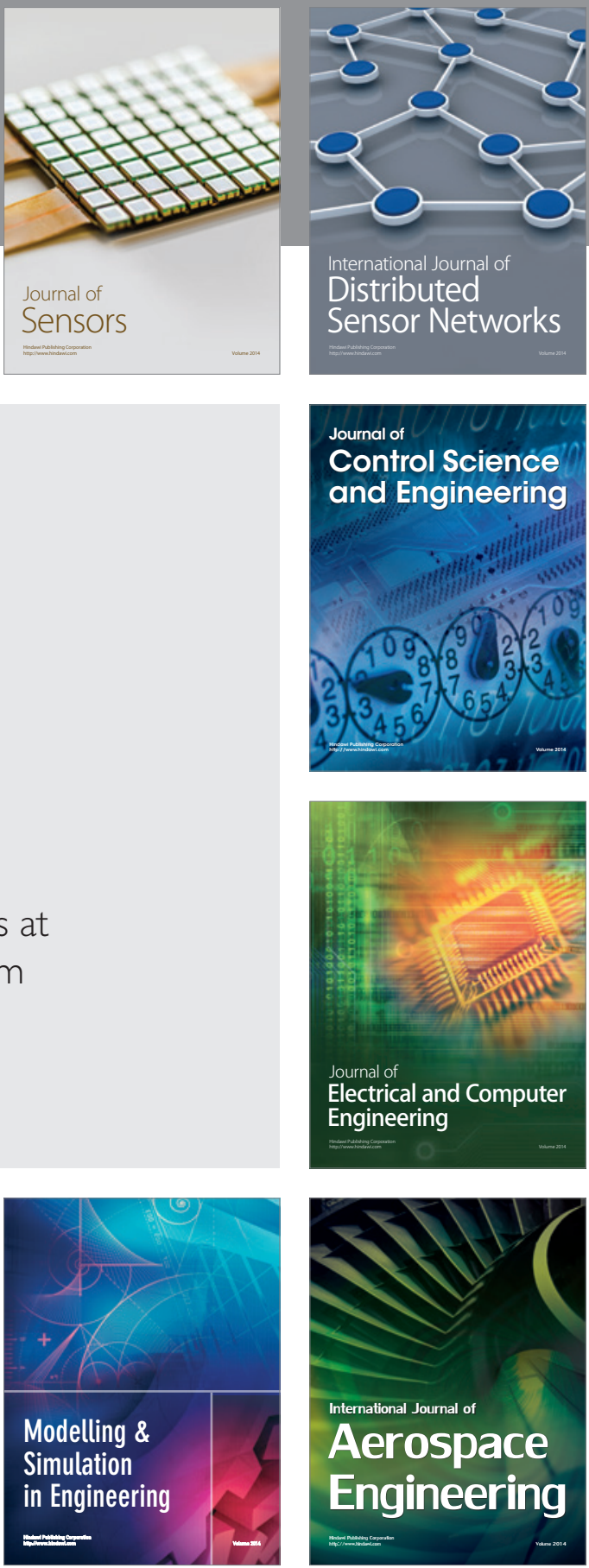

Journal of

Control Science

and Engineering
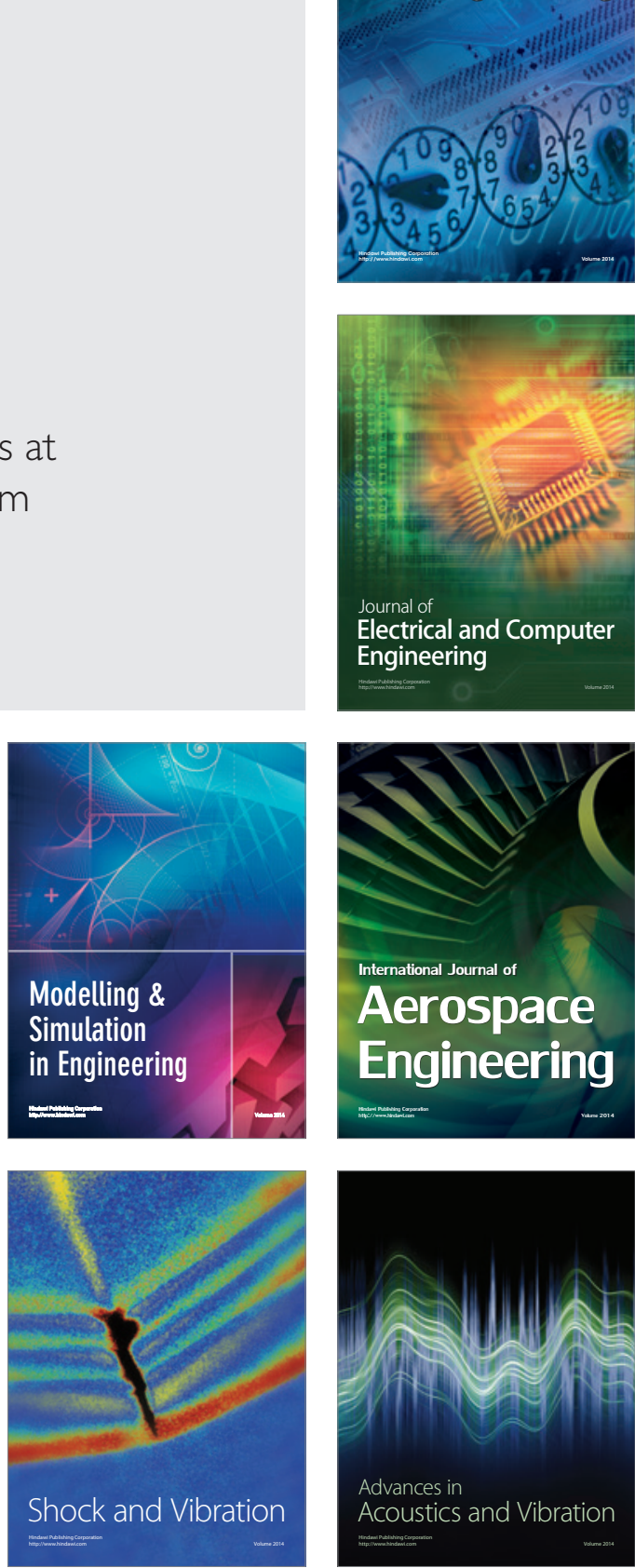